\title{
Modeling average gas-solid heat transfer using particle-resolved direct numerical simulation
}

\author{
B. Sun ${ }^{\mathrm{a}}$, S. Tenneti ${ }^{\mathrm{a}}$, S. Subramaniam ${ }^{\mathrm{a}, *}$ \\ ${ }^{a}$ Department of Mechanical Engineering, Center for Multiphase Flow Research, Iowa State \\ University,Ames, IA 50011, USA
}

\begin{abstract}
The purpose of this work is to develop gas-solid heat transfer models using Particle-resolved Direct Numerical Simulations (PR-DNS). Gas-solid heat transfer in steady flow through a homogeneous fixed assembly of monodisperse spherical particles is simulated using the Particle-resolved Uncontaminated-fluid Reconcilable Immersed Boundary Method (PUReIBM). PR-DNS results are obtained over a range of mean slip Reynolds number (1-100) and solid volume fraction (0.1-0.5). Fluid heating is important in gas-solid heat transfer, especially in dense low-speed flows, and the PUReIBM formulation accounts for this through a heat ratio which appears in the thermal self-similarity boundary condition that ensures thermally fully developed flow. The average volumetric interphase heat transfer rate (average gas-solid heat transfer) that appears in the average fluid temperature evolution equation is quantified and modeled using PR-DNS results. The Nusselt number corresponding to average gas-solid heat transfer is obtained from PR-DNS data, and compared with Gunn's Nusselt number correlation [1]. A new Nusselt number correlation is proposed that fits the PR-DNS data more closely and also captures the Reynolds number dependence more accurately. It is shown that the use of Nusselt number correlations based on the average bulk fluid temperature in the standard two-fluid model for gas-solid heat transfer is inconsistent, and results in up to $35 \%$ error in prediction of the average gas-solid heat transfer. Using PR-DNS data, a consistent two-fluid model is proposed that improves the predicted average gas-solid heat transfer.
\end{abstract}

Keywords: Gas-solid heat transfer, Particle-resolved direct numerical simulation, Average fluid temperature equation, Two-fluid model, Fixed

\footnotetext{
${ }^{*}$ Corresponding author

Email address: shankar@iastate.edu (S. Subramaniam)
} 


\section{Introduction}

Gas-solid heat transfer is important in many emerging technologies such as carbon-neutral energy generation using biomass [2], chemical looping combustion [3], and $\mathrm{CO}_{2}$ capture [4, 5, 6]. An improved understanding of gas-solid heat transfer is crucial for process and component design in the development of these technologies. CFD simulations $[7,8,9]$ of multiphase flow are increasingly being used as an efficient alternative to experiments for process and design optimization, because experiments are often costly and time-consuming. Since the averaged equations governing mass, momentum, and energy that are solved in multiphase CFD simulations are obtained by using a statistical averaging procedure [10,11], terms such as the average interphase transfer of momentum and energy between different phases need to be modeled.

Specifically, in the absence of mass transfer between phases the average fluid temperature equation from two-fluid theory $[7,12]$ reads as follows:

$$
\underbrace{\frac{\partial}{\partial t}\left\{\rho_{f} \varepsilon_{f} c_{p f}\left\langle T^{(f)}\right\rangle\right\}}+\underbrace{\frac{\partial}{\partial x_{j}}\left\{\rho_{f} \varepsilon_{f} c_{p f}\left\langle u_{j}^{(f)}\right\rangle\left\langle T^{(f)}\right\rangle\right\}}=\underbrace{\left\langle\frac{\partial I_{f}}{\partial x_{j}} q_{j}\right\rangle}
$$

unsteady term mean flow convection

(1) average gas-solid

$$
-\underbrace{\frac{\partial}{\partial x_{j}}\left\langle I_{f} q_{j}\right\rangle}
$$
heat transfer

(2) average conduction in the fluid phase

$$
-\underbrace{\frac{\partial}{\partial x_{j}}\left\{\rho_{f} c_{p f}\left\langle I_{f} u_{j}^{\prime \prime(f)} T^{\prime \prime(f)}\right\rangle\right\}}
$$

(3) transport of temperaturevelocity covariance

and it contains the following unclosed terms:

(1) average gas-solid heat transfer,

(2) average conduction in the fluid phase, and

(3) transport of temperature-velocity covariance.

In Eq. $1, \rho_{f}$ and $c_{p f}$ are the density and specific heat of the fluid phase, respectively, $q_{j}=-k_{f} \partial T / \partial x_{j}$ is the heat flux vector and $\varepsilon_{f}=\left\langle I_{f}\right\rangle$ is the volume fraction of the fluid phase, where $I_{f}(\mathrm{x}, t)$ is the fluid-phase indicator function that is unity if the point $\mathbf{x}$ lies on the fluid-phase at time $t$, and zero otherwise. If $\psi(\mathbf{x}, t)$ is any field (velocity or temperature), then its phasic average 
$\left\langle\psi^{(f)}\right\rangle(\mathbf{x}, t)$ (average fluid velocity $\left\langle u_{j}^{(f)}\right\rangle$ and average fluid temperature $\left\langle T^{(f)}\right\rangle$ ) is its average value conditional on being in the fluid phase, which is defined as:

$$
\left\langle\psi^{(f)}\right\rangle(\mathbf{x}, t)=\frac{\left\langle I_{f}(\mathbf{x}, t) \psi(\mathbf{x}, t)\right\rangle}{\left\langle I_{f}(\mathbf{x}, t)\right\rangle} .
$$

We use angle brackets to denote ensemble-averaging of random fields over all particle configurations, and an overbar to indicate spatial averages ${ }^{1}$ Using the phasic average, the fluctuating components of the fluid velocity and temperature in Eq. 1 are defined as $u_{j}^{\prime \prime(f)}=u_{j}-\left\langle u_{j}^{(f)}\right\rangle$ and $T^{\prime \prime(f)}=T-\left\langle T^{(f)}\right\rangle$, where these fluctuations depend on spatial location and time, although for brevity this dependence is not explicitly shown. The average fluid velocity is obtained by solving the averaged momentum and mass conservation equations. In order to solve Eq. 1 for the average fluid temperature, closure models are needed for terms (1)-(3). In a typical two-fluid simulation of gas-solid flow, this equation is coupled to a similar averaged temperature equation for the solid phase [13], but this work only focuses on models for average gas-solid heat transfer term in the average fluid temperature equation.

Although there has been theoretical analysis and experimental measurements of gas-solid heat transfer, it is difficult to develop models for the unclosed terms that are valid over a wide range of solid volume fraction and mean slip Reynolds number using these approaches. For instance, theoretical studies of heat transfer in gas-solid flow are limited to creeping flow past an isolated sphere [14], ordered spheres [15, 16], or moderate flow past random assemblies of spheres $[1,17]$. At finite Reynolds number, the nonlinearity of the governing equations and the randomness in particle positions and velocities pose significant obstacles to theoretical analysis. Experimental measurement of gas-solid heat transfer is also challenging because of limited optical access. Various experimental techniques, such as axial heat conduction [18], step response [19], frequency response [20,21], and shot response [22, 23] have been used to study heat transfer in gas-solid fixed-bed reactors over the last several decades [24]. However, these experimental studies report values for the Nusselt number in packed beds $\left(\varepsilon_{s} \approx 0.6\right)$ that differ by three or four orders of magnitude [24]. Since most experimental measurements of heat transfer in gas-solid flow are in-

\footnotetext{
${ }^{1}$ For this problem these spatial averages appear as either a cross-sectional average of a random field that still depends on the particle configuration, or as a streamwise average of an inhomogeneous ensemble-averaged field.
} 
trusive, the flow disturbance caused by the probes is also a source of uncertainty. Furthermore, the gas-solid heat transfer rate is inferred from point-wise temperature measurements using simplified one-dimensional models of heat transfer that are based on assumptions such as the neglect of axial conduction in the fluid phase. Therefore, the validity of the assumptions implicit in these simplified one-dimensional models used in the inferential procedure is also a source of uncertainty.

This motivates us to use particle-resolved direct numerical simulation (PRDNS), which is a first-principles, model-free simulation method that solves for the instantaneous three-dimensional velocity and temperature fields representing the flow and heat transfer around each particle. PR-DNS can be used to quantify the unclosed terms in Eq. 1, since these terms can be directly calculated from the instantaneous three-dimensional velocity and temperature fields. Indeed, several researchers have successfully extracted correlations for the average interphase momentum transfer in gas-solid flow by simulating steady flow past statistically homogeneous fixed assemblies of spherical particles $[25,26,27,28,29,30]$ in periodic domains. In recent years, researchers have also used PR-DNS to investigate heat transfer in gas-solid flow. Twodimensional PR-DNS studies [31, 32] on simulating gas-solid flow in an infinite channel with an isothermal particle have been reported. Haeri and Shrimpton [33] set up a staggered tube bank to study convective heat transfer in gas-solid flow with inflow and outflow boundary conditions. Massol and Simonin [34] studied heat transfer in a fixed array of monodisperse spheres using three-dimensional PR-DNS. However, their formulation uses a source/sink term that is strictly valid only for those cases where fluid heating is negligible during the time it takes the flow to transit the computational domain. This restriction holds only for dilute suspensions at high Reynolds number [17]. Deen et al. $[35,36]$ and Tavassoli et al. [37] used three-dimensional PR-DNS with inflow and outflow boundary conditions to compute the gas-solid heat transfer coefficient and Nusselt number that are compared with Gunn's correlation [1]. Feng and Musong [38] found that the entrance effect of heat transfer on particles in the fluidized bed is to produce high heat transfer rate on particles. However, these studies did not quantify all the unclosed terms in the average fluid temperature equation (Eq. 1).

Recently, Tenneti et al. [39] have shown that fluid heating can be accounted for in three-dimensional PR-DNS of thermally fully-developed flow in periodic domains using a thermal self-similarity condition. In that work, the role of fluid 
heating and the Nusselt number for gas-solid heat transfer was reported for a limited range of Reynolds number $\operatorname{Re}_{\mathrm{m}}$ and volume fraction $\varepsilon_{s}$. This study builds on the work of Tenneti et al. [39] to comprehensively characterize the dependence of Nusselt number on solid volume fraction and mean slip Reynolds number, and to quantify and model the average gas-solid heat transfer.

Gunn [1] developed a Nusselt number correlation for average gas-solid heat transfer that is a fit to experimental data from many sources that vary by several orders of magnitude [24]. Gunn's Nusselt number correlation can be used to compute the average gas-solid heat transfer, and it is widely used to model the first unclosed term of the right-hand side (RHS) of Eq. 1 (average gas-solid heat transfer) in CFD simulation of gas-solid heat transfer [40]. Tavassoli et al. [37] and Tenneti et al. [39] have used PR-DNS to quantify the average Nusselt number to compare with Gunn's correlation over a limited range of Reynolds number and volume fraction. However, the performance of Gunn's correlation needs to be assessed over a wide range of these parameters using PR-DNS. Additionally, the Nusselt number is computed in terms of the average bulk fluid temperature in Gunn's correlation [1] and in the PR-DNS by Tenneti et al. [39] and Tavassoli et al. [37]. However, in the two-fluid model, the difference between average fluid temperature (instead of the average bulk fluid temperature) and average solid temperature is used to compute the average gas-solid heat transfer. We address the question whether using the average fluid temperature in the twofluid model is consistent with the Nusselt number definition, and whether this model is appropriate to accurately compute the gas-solid heat transfer.

The rest of the paper is organized as follows. In Section 2, we describe the heat transfer problem in a fixed particle assembly and discuss the assumptions needed to simplify this problem. In Section 3, the PR-DNS approach that is used to solve this heat transfer problem is briefly described. The computation of the unclosed average gas-solid heat transfer term in the two-fluid model from PR-DNS data is then described. In Section 4, numerical convergence and the choice of numerical parameters are discussed. In Section 5 we compute the average Nusselt number and propose an new Nusselt number correlation for gassolid heat transfer using PR-DNS data. We evaluate the accuracy of a standard two-fluid model for the average gas-solid heat transfer rate by comparison with PR-DNS data and propose an improved model in Section 6 . Finally, Section 7 discusses the applicability of the results and in Section 8 we summarize the principal findings of this work. 


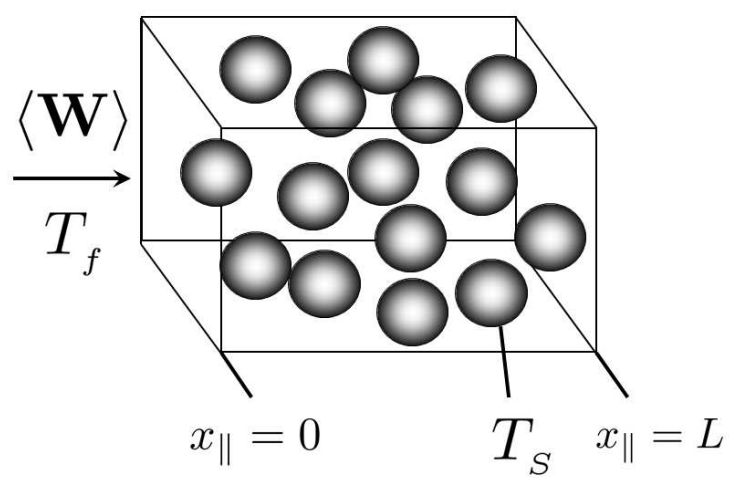

Figure 1: Schematic of steady flow with mean slip velocity $\langle\mathbf{W}\rangle$ through a fixed assembly of isothermal particles. The temperatures in the fluid phase and solid phase are $T_{f}(\mathbf{x}, t)$ and $T_{s}$, respectively. The inlet and outlet of the domain are at $x_{\|}=0$ and $x_{\|}=L$, respectively.

\section{Gas-solid heat transfer in flow past a fixed assembly of particles}

We consider gas-solid heat transfer in a steady flow past a homogeneous fixed assembly of monodisperse spherical particles as Fig. 1 shows. Although the hydrodynamic problem is statistically homogeneous, the mean fluid velocity is anisotropic. This directionality in the flow implies that fluid that is downstream of a particle is heated up (or cooled down) by interphase heat transfer, relative to fluid that is upstream of the particle. In other words, when we have forced convection heat transfer in a fixed assembly of particles with finite mean slip velocity $\langle\mathbf{W}\rangle=\left\langle\mathbf{u}^{(f)}\right\rangle-\left\langle\mathbf{u}^{(s)}\right\rangle$, where $\left\langle\mathbf{u}^{(f)}\right\rangle$ and $\left\langle\mathbf{u}^{(s)}\right\rangle$ are the average velocities in the fluid phase and solid phase, the fluid temperature varies with streamwise location in the particle assembly, and the average fluid temperature cannot be assumed to be uniform. With this heating or cooling of fluid by particles, the thermal problem becomes statistically inhomogeneous in the streamwise direction. This feature of heat transfer in gas-solid flows is well established [17].

The inhomogeneity of the fluid temperature in a fixed particle assembly implies that in order to model unclosed terms in the average fluid temperature equation (Eq. 1), the average gas-solid heat transfer needs to be extracted from spatially varying surface statistics. However, spatially varying surface statistics converge slowly [41] even with a large number of realizations, where 
each realization corresponds to a different particle configuration with the same solid volume fraction and pair correlation function. However, Tenneti et al. [39] showed that if the flow is thermally fully-developed, then the Nusselt number is statistically homogeneous even though the average fluid temperature varies in the streamwise direction. Therefore, in a thermally fully-developed flow, the average Nusselt number can be computed by volume-averaging using fewer realizations. Tenneti et al. [39] developed a thermal self-similarity condition for gas-solid heat transfer in steady flow past a statistically homogeneous fixed assembly of particles that results in a thermally fully-developed flow. The same boundary condition has also been used by Tyagi \& Acharya [42] for simulating heat transfer in duct flow. We briefly summarize Tenneti et al.'s [39] formulation of thermally fully-developed gas-solid flow here for completeness.

The assumptions made to simplify this heat transfer problem are the same as in Tenneti et al. [39, 43], namely: (i) isothermal particles with a single spatially uniform temperature for all particles that is constant in time, and (ii) neglect of radiation and free convection. A detailed justification for these assumptions can be found in Tenneti et al. [39]. Constant gas properties are also assumed for simplicity of the computation. The fluid temperature field $T(\mathbf{x}, t)$, in the absence of viscous heating, radiation and free convection effects, obeys the following convection-diffusion equation:

$$
\frac{\partial T}{\partial t}+\frac{\partial\left(u_{j} T\right)}{\partial x_{j}}=\alpha_{f} \frac{\partial^{2} T}{\partial x_{j} \partial x_{j}},
$$

where $\alpha_{f}=k_{f} / \rho_{f} c_{P f}$ is the thermal diffusivity in the fluid phase, and $k_{f}$ is the thermal conductivity in the fluid phase. Equation (3) needs to be solved in the fluid with the Dirichlet boundary condition of $T=T_{s}$ at the surface of the particles, where $T_{s}$ is the uniform temperature at which all the particles are maintained. As noted earlier, the average fluid temperature varies in the streamwise direction due to fluid heating (or cooling) by the particles. Since interphase heat transfer is driven by the difference between the temperatures in the solid and fluid phases, this results in an average interphase heat flux that also varies along the streamwise direction. The ratio of the response (average heat flux) to the driving force (average temperature difference) determines the Nusselt number, that in general also varies along the streamwise direction. However, if the flow is thermally fully-developed (as in internal pipe flow, see [44] 
for example), then the locally scaled excess fluid temperature field ${ }^{2} \theta$, defined as:

$$
\theta(\mathbf{x}, t)=\frac{T(\mathbf{x}, t)-T_{s}}{\left\langle T_{m}\right\rangle\left(x_{\|}, t\right)-T_{s}},
$$

is statistically homogeneous at steady state and does not vary in the streamwise or axial direction $x_{\|}[39]$, i.e.,

$$
\frac{\partial \theta}{\partial x_{\|}}=\frac{\partial}{\partial x_{\|}}\left(\frac{T(\mathbf{x})-T_{s}}{\left\langle T_{m}\right\rangle\left(x_{\|}\right)-T_{s}}\right)=0 .
$$

In the above definition, $\left\langle T_{m}\right\rangle\left(x_{\|}, t\right)$ is the ensemble-averaged bulk fluid temperature or "mixing-cup" temperature, which is defined as the average of the bulk fluid temperature on each realization $\omega$ (corresponding to a particle configuration, which occurs with probability $\left.d P_{\omega}\right)$, such that

$$
\left\langle T_{m}\right\rangle\left(x_{\|}, t\right)=\int_{\omega \in \Omega} T_{m}\left(x_{\|}, t ; \omega\right) d P_{\omega},
$$

where the bulk fluid temperature on each realization is

$$
T_{m}\left(x_{\|}, t ; \omega\right)=\frac{\int_{A_{f}}(\mathbf{u} T) \cdot \mathbf{e}_{\|} d A_{f}}{\int_{A_{f}} \mathbf{u} \cdot \mathbf{e}_{\|} d A_{f}},
$$

where $\mathbf{e}_{\|}$is the unit vector along the streamwise direction and $A_{f}$ is the area occupied by the fluid in a plane perpendicular to the streamwise direction. In general for any function $Q\left(x_{\|}, t ; \omega\right)$ that is defined for a realization $\omega$, we define the ensemble-average as

$$
\langle Q\rangle\left(x_{\|}, t\right)=\int_{\omega \in \Omega} Q\left(x_{\|}, t ; \omega\right) d P_{\omega} .
$$

The thermally fully-developed condition implies that at steady state the local wall heat flux scaled by the temperature difference $\left(\left\langle T_{m}\right\rangle\left(x_{\|}\right)-T_{s}\right)$ is a constant. In other words, the spatial variation of interphase heat flux in response to the temperature difference occurs in such a way that the heat transfer coefficient (or the Nusselt number) at each axial location remains the same throughout the bed. The advantage of establishing a thermally fully-developed flow is that there are no entrance length effects and the average Nusselt number can be calculated by averaging over all the particles in the bed, rather than accounting for

\footnotetext{
${ }^{2}$ For simplicity this quantity is later referred to as simply the scaled fluid temperature.
} 
its variation in the streamwise direction. This is accomplished by implementing a thermal self-similarity condition, which requires periodic boundary conditions on the scaled fluid temperature [39].

For reasons detailed in Tenneti et al. [39], it is easier to transform the periodic boundary conditions on $\theta$ to obtain similarity conditions on the temperature field $T(\mathbf{x}, t)$ and solve Eq. 3 for $T(\mathbf{x}, t)$. Simplification of the thermal similarity conditions and homogenization of the boundary conditions on the particle surfaces is accomplished by defining a non-dimensional excess temperature field ${ }^{3}$ $\phi(\mathbf{x}, t)$ as follows:

$$
\phi(\mathbf{x}, t)=\frac{T(\mathbf{x}, t)-T_{s}}{\left\langle T_{m, i n}\right\rangle-T_{s}},
$$

where $\left\langle T_{m, i n}\right\rangle$ is the average inlet bulk fluid temperature that is defined by Eq. 6 in terms of the inlet bulk fluid temperature $T_{m, i n}$, which is given by Eq. 7 evaluated at $x_{\|}=0$. Using this definition of the non-dimensional temperature, it is easy to see that the average non-dimensional bulk fluid temperature $\left\langle\phi_{m}\right\rangle$ has a similar definition:

$$
\left\langle\phi_{m}\right\rangle\left(x_{\|}, t\right)=\frac{\left\langle T_{m}\right\rangle\left(x_{\|}, t\right)-T_{s}}{\left\langle T_{m, i n}\right\rangle-T_{s}} .
$$

Substituting Eq. 9 in Eq. 3 gives the governing equation for the non-dimensional temperature:

$$
\frac{\partial \phi}{\partial t}+\frac{\partial\left(u_{j} \phi\right)}{\partial x_{j}}=\alpha_{f} \frac{\partial^{2} \phi}{\partial x_{j}^{2}} .
$$

The isothermal boundary conditions on the particle surface reduce to $\phi=0$. The periodic boundary conditions on $\phi$ now appear in a very simple form:

$$
\begin{aligned}
\phi(0, y, z) & =r_{h} \phi(L, y, z), \\
\phi\left(x_{\|}, 0, z\right) & =\phi\left(x_{\|}, L, z\right), \\
\phi\left(x_{\|}, y, 0\right) & =\phi\left(x_{\|}, y, L\right),
\end{aligned}
$$

where $r_{h}$ is the heat ratio, which is defined as:

$$
r_{h}=\frac{\left\langle T_{m, \text { in }}\right\rangle-T_{s}}{\left\langle T_{m, \text { out }}\right\rangle-T_{s}} .
$$

In this definition of the heat ratio $\left\langle T_{m, \text { out }}\right\rangle$ is the average bulk fluid temperature at $x_{\|}=L$, and $L$ is the length of the box. The heat ratio is the ratio of the

\footnotetext{
${ }^{3}$ For simplicity this quantity is referred to as the non-dimensional temperature.
} 
excess bulk fluid temperature at the inlet $\left(x_{\|}=0\right)$ to the excess bulk fluid temperature at the outlet $\left(x_{\|}=L\right)$. In other words, the heat ratio is simply the inverse of the average non-dimensional bulk fluid temperature at $x_{\|}=L$ i.e.,

$$
r_{h}=\frac{1}{\left\langle\phi_{m}\right\rangle_{\text {out }}} .
$$

The heat ratio quantifies by how much a fluid particle heats up when it leaves the box and so this quantity depends solely on the flow structure and the interphase heat transfer in the domain. Note that the heat ratio, or the amount by which the fluid gets heated up (or cooled down) when it reaches the end of the box, is an unknown quantity and is obtained as a part of the solution.

There is a useful relation that shows that the non-dimensional temperature (Eq. 9) is simply the product of the scaled temperature (Eq. 4) and the average non-dimensional bulk fluid temperature (Eq. 10):

$$
\phi(\mathbf{x}, t)=\left(\frac{T(\mathbf{x}, t)-T_{s}}{\left\langle T_{m}\right\rangle\left(x_{\|}, t\right)-T_{s}}\right)\left(\frac{\left\langle T_{m}\right\rangle\left(x_{\|}, t\right)-T_{s}}{\left\langle T_{m, i n}\right\rangle-T_{s}}\right)=\theta(\mathbf{x}, t)\left\langle\phi_{m}\right\rangle\left(x_{\|}, t\right) .
$$

Multiplying the above equation by the fluid indicator function $I_{f}$, taking the expectation (see Eq. 8), and using the definition in Eq. 2 leads to the corresponding relation between the phase-averaged counterparts:

$$
\left\langle\phi^{(f)}\right\rangle(\mathbf{x}, t)=\left\langle\theta^{(f)}\right\rangle(\mathbf{x}, t)\left\langle\phi_{m}\right\rangle\left(x_{\|}, t\right),
$$

Also noting that the $\theta$ field is statistically homogeneous at steady state reveals that the inhomogeneity in the steady average fluid temperature field arises solely from the inhomogeneity in the bulk fluid temperature:

$$
\left\langle\phi^{(f)}\right\rangle\left(x_{\|}\right)=\left\langle\theta^{(f)}\right\rangle\left\langle\phi_{m}\left(x_{\|}\right)\right\rangle .
$$

In the next section we describe the numerical method that is used to solve the heat transfer problem and extract unclosed terms.

\section{Numerical method}

The gas-solid heat transfer problem described in Sec. 2 can be solved using our PR-DNS approach, which is called the Particle-resolved Uncontaminatedfluid Reconcilable Immersed Boundary Method (PUReIBM) [30, 43, 45]. 


\subsection{PUReIBM formulation}

Complete details of the PUReIBM hydrodynamic solver are discussed by Tenneti et al. [30, 45, 46] and Garg et al. [47]. The extension of the PUReIBM hydrodynamic solver to account for the temperature equation in gas-solid heat transfer is described in Tenneti et al. [39]. Here we briefly review the numerical approach to solve the gas-solid heat transfer problem in a fixed assembly of isothermal particles.

It is worth noting that the equations in Section 2 are formulated in terms of the ensemble-averaged bulk fluid temperature (see Eqs. 9 and 13). The solution to these equations can be accomplished by simultaneously solving Eq. 11 in parallel for several different particle configurations subject to the boundary conditions is Eqs. 12 and 14 on a parallel computer. In this setup each particle configuration and corresponding fluid temperature field is stored on a node, and the ensemble-averaged bulk fluid temperature is communicated to all nodes at the end of each time step. However, it turns out that the statistical variability of the bulk fluid temperature and heating ratio in different particle configurations is small, provided the computational domains are sufficiently large. Therefore, our approach has been to replace the ensemble-averaged bulk fluid temperature with the bulk fluid temperature in that realization. In this case the scaled fluid temperature for each realization is rewritten as follows,

$$
\theta(\mathbf{x}, t ; \omega)=\frac{T(\mathbf{x}, t ; \omega)-T_{s}}{T_{m}\left(x_{\|}, t ; \omega\right)-T_{s}},
$$

and the non-dimensional fluid temperature is rewritten as

$$
\phi(\mathbf{x}, t ; \omega)=\frac{T(\mathbf{x}, t ; \omega)-T_{s}}{T_{m, i n}(\omega)-T_{s}}
$$

This effectively decouples the temperature solution in different particle configurations and allows the solution in each realization to proceed independently. Ensemble-averaged quantities are computed from the individual steady temperature fields corresponding to each realization, as described in the next section. The small statistical variability in the bulk fluid temperature and heating ratio from one realization to another justify this decoupling approach. We can use Eqs. 18 and 19 at steady state to infer the following useful relation between the non-dimensional, scaled and bulk fluid temperature fields from each realization, 
which is the analog of Eq. 15:

$$
\phi(\mathbf{x} ; \omega)=\left(\frac{T(\mathbf{x} ; \omega)-T_{s}}{T_{m}\left(x_{\|} ; \omega\right)-T_{s}}\right)\left(\frac{T_{m}\left(x_{\|} ; \omega\right)-T_{s}}{T_{m, i n}(\omega)-T_{s}}\right)=\theta(\mathbf{x} ; \omega) \phi_{m}\left(x_{\|} ; \omega\right) .
$$

In PUReIBM [39], the following non-dimensional fluid temperature equation is solved at all grid nodes

$$
\rho_{f} c_{p f}\left[\frac{\partial \phi}{\partial t}+\frac{\partial\left(u_{j} \phi\right)}{\partial x_{j}}\right]=-\frac{\partial q_{j}^{\phi}}{\partial x_{j}}+I_{s} f_{\phi},
$$

where $q_{j}^{\phi}=-k_{f} \partial \phi / \partial x_{j}$ is the heat flux per unit temperature difference, $I_{s}$ is the solid-phase indicator function, and $f_{\phi}$ is the scalar Immersed Boundary (IB) direct forcing in the solid phase. The immersed boundary forcing accounts for the presence of the solid particles in the domain by ensuring that the isothermal boundary condition $\phi=0$ is satisfied on the surface of each solid particle. The surface of the solid particle is represented by a discrete number of points called boundary points. For spherical particles, the boundary points are specified by discretizing the sphere in spherical coordinates. Another set of points called exterior points are generated by projecting these boundary points onto a sphere of radius $r+\Delta r$, where $r$ is the radius of the particle. Similarly, the boundary points are projected onto a smaller sphere of radius $r-\Delta r$ and these points are called interior points. In our simulations $\Delta r$ is taken to be same as the grid spacing. The IB forcing is computed only at the interior points. At these points the fluid temperature is forced in a manner similar to the ghost cell approach used in standard finite-difference/finite-volume based methods [48]. For the boundary condition $\phi=0$ used in this work, the value of $\phi$ at the interior points is forced to be opposite in magnitude to the value of $\phi$ at the corresponding exterior points.

A distinctive feature of PUReIBM is that the scalar IB forcing $f_{\phi}$ is computed only at points lying inside the solid particles. This ensures that the fluid-phase temperature field is not contaminated by the scalar IB forcing term $f_{\phi}$, just as the fluid-phase velocity field is not contaminated by the hydrodynamic IB forcing. The consequences of fluid velocity contamination by IB forcing are discussed in detail by Tenneti et al. [30]. The computation of $f_{\phi}$ is similar to the computation of the IB forcing for the velocity field. The scalar IB forcing at the $(n+1)$ th time-step $f_{\phi}^{n+1}$ is specified to cancel the remaining terms in the governing equation and forces the non-dimensional temperature $\phi^{n}$ to its 
desired value $\phi^{d}$ at the particle surface:

$$
f_{\phi}^{n+1}=\rho_{f} c_{p f} \frac{\phi^{d}-\phi^{n}}{\Delta t}+\rho_{f} c_{p f} C_{\phi}^{n}+\left(\frac{\partial q_{j}^{\phi}}{\partial x_{j}}\right)^{n}
$$

where $C_{\phi}^{n}=\left[\partial\left(u_{j} \phi\right) / \partial x_{j}\right]^{n}$ is the convective term at the $n$th time-step. The non-dimensional fluid temperature equation (Eq. 21) is solved using a pseudospectral method, with the Crank-Nicolson scheme for the viscous terms, and an Adams-Bashforth scheme for the convective terms. Fourier transforms are used in the cross-stream directions and a finite-difference scheme is used in the streamwise direction [49]. Implementation of the periodic boundary conditions in Eq. 12 results in an independent set of cyclic tridiagonal systems that are solved using the Sherman-Morrison formula [45]. Details on the numerical method can be found elsewhere [30, 39, 45, 47]. A noteworthy feature of this approach is that the heat ratio $r_{h}$ in Eq. 12 is an unknown quantity and is solved iteratively. The temperature field is initialized with $r_{h}=1$ and the simulation is marched in pseudo time until the value of the heat ratio converges.

\subsection{Relation of PR-DNS data to unclosed terms}

In order to quantify the unclosed terms in the average fluid temperature equation in Eq. 1 from PR-DNS data, the correspondence between PR-DNS data and the unclosed terms needs to be established. The thermal fullydeveloped condition guarantees that terms like the Nusselt number and the scaled fluid temperature $\theta$ are statistically independent of the streamwise direction as shown by Tenneti et al. [39]. Therefore, we use volume-averaging over the fluid domain to compute those terms. However, since the average fluid temperature varies along the streamwise direction, terms like the average gas-solid heat transfer are not statistically homogeneous and they depend on the streamwise direction. For such terms we use a cross-sectional average, for instance, the average gas-solid heat transfer is computed from the local volumetric interphase heat transfer rate. In order to define the local volumetric gas-solid heat transfer rate $q_{\phi}^{\prime \prime \prime}\left(x_{\|} ; \omega\right)$ (see Eq. 37), the PR-DNS instantaneous fluid temperature equation (Eq. 1) is integrated over the cross-sectional area of fluid region $A_{f}$ in the $y-z$ plane perpendicular to the streamwise direction $x_{\|}$(see details in Appendix A). Cross-sectionally averaged terms such as the local volumetric interphase heat transfer rate $q_{\phi}^{\prime \prime \prime}\left(x_{\|} ; \omega\right)$ only depend on the streamwise coordinate. 
For cross-sectionally averaged terms such as the local volumetric interphase heat transfer rate, the numerical value from PR-DNS data for every realization $\omega$ would equal the corresponding average in the limit that the cross-sectional area of the domain tends to infinity. In practice, acceptable convergence can be obtained with large domain sizes, but the domain size is limited by computer memory. However, even domains that are large enough such that two-point correlations decay to zero may not provide good particle statistics especially at low solid volume fraction. However, the statistical variability in the PRDNS estimate of the unclosed terms arising from finite number of particles in each domain can be reduced by averaging over multiple independent simulations (MIS) or realizations. For instance, the average gas-solid heat transfer term is estimated from PR-DNS data as

$$
\left\langle\frac{\partial I_{f}}{\partial x_{j}} q_{j}^{\phi}\right\rangle\left(x_{\|}\right) \approx \frac{1}{M} \sum_{\omega=1}^{M}\left\{q_{\phi}^{\prime \prime \prime}\left(x_{\|} ; \omega\right)\right\}
$$

where $q_{\phi}^{\prime \prime \prime}\left(x_{\|} ; \omega\right)$ is given by Eq. 37 (see Appendix A for details) and $M$ is the number of realizations. The choice of number of MIS depends on the statistic being estimated. We find that mean values and second moments converge fairly quickly within 4 or 5 realizations. Higher-order statistics would require a larger number of realizations to reduce statistical variability.

\section{Simulation Results and Numerical Convergence}

We have performed PR-DNS simulations over a range of mean slip Reynolds number $\operatorname{Re}_{\mathrm{m}}=1-100$ and solid volume fraction $\varepsilon_{s}=0.1-0.5$ in gas-solid flow with Prandtl number of 0.7 , as summarized in Table 1 . Before discussing the results, we establish numerical convergence of the simulation method for steady gas-solid heat transfer in a fixed particle assembly.

\subsection{Numerical convergence}

Numerical convergence and accuracy of the scalar solver in PUReIBM have been established in Tenneti et al. [39]. The principal numerical parameters relevant to this study are the grid resolution $D_{m}=D / \Delta x$, domain size $L / D$ and the number of independent simulations. Since these are steady calculations, the time-marching is done in pseudo-time. The Courant-Friedrich-Lewy (CFL) number based on mean slip velocity and grid size is always less than 0.5. In a previous study [47] we have shown that the code is stable for this choice of CFL number, and yields convergent solutions with this choice of pseudo-time step. 


\begin{tabular}{ccccc}
\hline$\varepsilon_{s}$ & $\operatorname{Re}_{m}$ & $L / D$ & $N_{p}$ & $D_{m}$ \\
\hline 0.1 & $1,5,10,20,30,40,50,100$ & 7.5 & 80 & 20 \\
0.2 & $1,5,10,20,30,40,50,100$ & 7.5 & 161 & 20 \\
0.3 & $1,5,10,20,30,40,50,100$ & 5 & 71 & 30 \\
0.4 & $1,5,10,20,30,40,50,100$ & 5 & 95 & 30 \\
0.5 & $1,5,10,20,30,40,50,100$ & 4 & 61 & 40 \\
\hline
\end{tabular}

Table 1: Parameters for simulation of heat transfer in steady flow past random fixed assemblies of particles. The physical parameters are the solid volume fraction $\varepsilon_{s}$ and the mean slip Reynolds number $\operatorname{Re}_{\mathrm{m}}$. The numerical parameters are the ratio of the box length to the particle diameter $L / D$ and the grid resolution $D_{m}=D / \Delta x$. The number of particles $N_{p}$ is determined by $\varepsilon_{s}$ and $L$. Five independent simulations of each case are simulated to reduce statistical variability.

\subsection{Grid resolution}

Tenneti et al. [39] compared PUReIBM simulations of steady heat transfer in a duct with an analytical result and showed that the simulation results converge accurately to the analytical value with increasing grid resolution. Here we demonstrate numerical convergence of the Nusselt number for steady heat transfer in flow past random assemblies of monodisperse spherical particles.

Figure 2(a) shows the convergence characteristics of the volumetric mean Nusselt number (see Eq. 43 in Appendix A) with respect to grid resolution $D_{m}=$ $D / \Delta x$, where $D$ is the particle diameter and $\Delta x$ is the grid spacing, We choose the box length to be $L / D=4$ so that different grid resolutions can be tested within available computational resources. Elsewhere [50] we have used the decay of scaled fluid temperature autocorrelation to justify the choice of box length. All simulations here are repeated with the same random particle configuration. The relative error in the volumetric mean Nusselt number between the coarsest grid $D_{m}=20$ and the finest grid $D_{m}=70$ is about $11 \%$. The volumetric mean Nusselt number at $\varepsilon_{s}=0.4$ and $\operatorname{Re}_{\mathrm{m}}=20$ also converges to an asymptotic value of 7.8 with increasing grid resolution.

\subsection{Number of realizations}

Besides the grid resolution, the statistical variability arising from finite number of particles can be an issue as discussed in Section 3.2. In order to obtain good statistics, we need to reduce the statistical variability in the PR-DNS estimate of the unclosed terms arising from finite number of particles by averaging over MIS corresponding to independent particle configurations. The choice of the number of MIS depends on the convergence of statistics as Figure 2(b) shows. This figure shows the convergence of average Nusselt number 


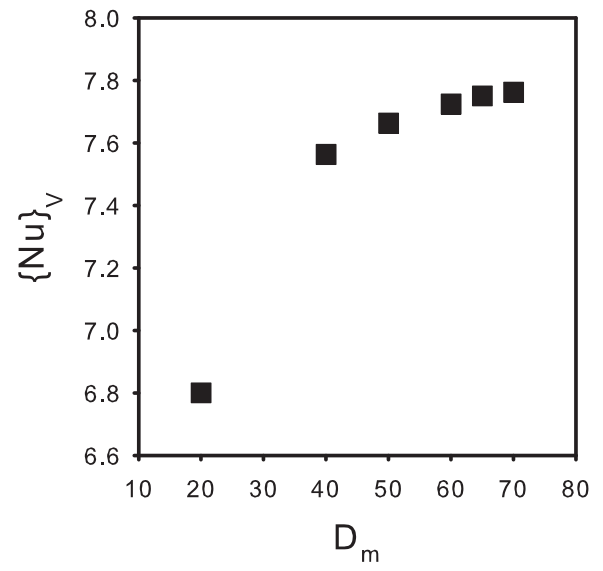

(a)

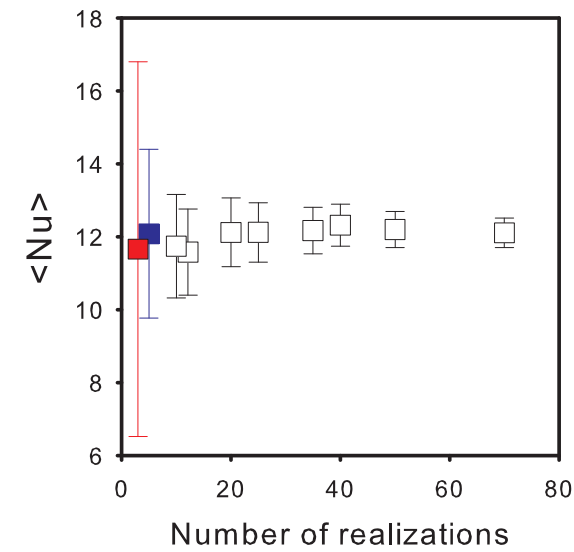

(b)

Figure 2: (a) Convergence characteristics of the volumetric mean Nusselt number (see Eq. 43) with grid resolution $D_{m}=D / \triangle x$ for heat transfer in a random assembly of spheres at $\operatorname{Re}_{\mathrm{m}}=20$ and $\varepsilon_{s}=0.4$. The same random particle configuration with $L / D=4$ is used for all grid resolution values. (b) Dependence of average Nusselt number on number of MIS or realizations at $\operatorname{Re}_{\mathrm{m}}=20$ and $\varepsilon_{s}=0.4$. Symbols indicate average Nusselt number and error bars indicate $95 \%$ confidence intervals. The red and blue filled symbol represent the average Nusselt number obtained using 2 and 5 realizations, respectively.

with increasing number of MIS at $\varepsilon_{s}=0.4$ and $\operatorname{Re}_{\mathrm{m}}=20$, along with the $95 \%$ confidence intervals corresponding to each set of MIS. We conclude that we can estimate the true average (obtained with 70 realizations) using 5 realizations, while recognizing that the $95 \%$ confidence intervals with 5 realizations correspond to about $15 \%$ of the true average value. Hence, we use 5 MIS for all simulations to obtain the average Nusselt number over a range of Reynolds number and volume fraction.

\subsection{Choice of numerical parameters}

In addition to the grid resolution and number of realizations, the box length is also a numerical parameter, and we have discussed its choice elsewhere [50]. In that work [50] it is shown that the scaled temperature autocorrelation decays within a box of length $5 D$ for a solid volume fraction of 0.4 and Reynolds number 100 (lower Reynolds number cases decay to zero within shorter separation distances). Based on this comprehensive study of the dependence of our PR-DNS heat transfer results on the principal numerical parameters, we have chosen values for grid resolution and number of independent simulations that minimize numerical error within the available computational resources (see Ta- 
ble 1). However, since we have explored a wide parameter range, each of these choices does incur some numerical error, and we estimate that in the worst case scenario where all the errors add cumulatively, the maximum numerical error in the average Nusselt number would be $15-20 \%$.

We can estimate the maximum numerical error using an error model similar to that Garg et al. [51] and Xu et al. [52] used. The total numerical error in the average Nusselt number is defined as

$$
\epsilon_{\mathrm{Nu}}=\{\mathrm{Nu}\}_{V, D_{m}}-\langle\mathrm{Nu}\rangle,
$$

where $\{\mathrm{Nu}\}_{V, D_{m}}$ is the numerical estimate. This total numerical error contains contributions from grid resolution and finite number of particles. In order to calculate the total numerical error in the average Nusselt number, the total numerical error $\epsilon_{\mathrm{Nu}}$ can be decomposed as

$$
\epsilon_{\mathrm{Nu}}=\Sigma_{\mathrm{Nu}}+D_{\mathrm{Nu}}=\Sigma_{\mathrm{Nu}}+B_{\mathrm{Nu}}+S_{\mathrm{Nu}},
$$

where $\Sigma_{\mathrm{Nu}}$ is the statistical error, and $D_{\mathrm{Nu}}$ is the deterministic error and is further decomposed into bias error $B_{\mathrm{Nu}}$ and discretization error $S_{\mathrm{Nu}}$. Note that it is infeasible to determine these coefficients for the entire parameter range of solid volume fraction and Reynolds number with current computational resources. Therefore, we construct the error model based on available PR-DNS data and apply it to find the maximum error in the worst case scenario that corresponds to the lowest grid resolution. Based on this error model, the total numerical error for the worst case scenario with grid resolution $D_{m}=20$ is $18.5 \%$, with the major contribution of $12 \%$ coming from the discretization error (statistical error contributes $4.5 \%$ and bias error contributes only $2 \%$ ). Of all the simulations, only two cases corresponding to $\varepsilon_{s}=0.1$ and $\varepsilon_{s}=0.2$ use a grid resolution $D_{m}=20$. All the other cases simulated use higher grid resolution $D_{m} \geq 30$ and therefore incur less discretization error, and consequently less total error. Note that the difference between existing correlations such as Gunn's correlation and the measured data (see Figure 3 ) is several orders of magnitude larger than this error. It is also worth noting that the total numerical error that we have estimated in our "worst case" scenario with $D_{m}=20$ is still low compared to the usual error associated with convection heat transfer correlations that is estimated at about 25\% (see Chapter 7.2.6 in Incropera et al. [44]). 


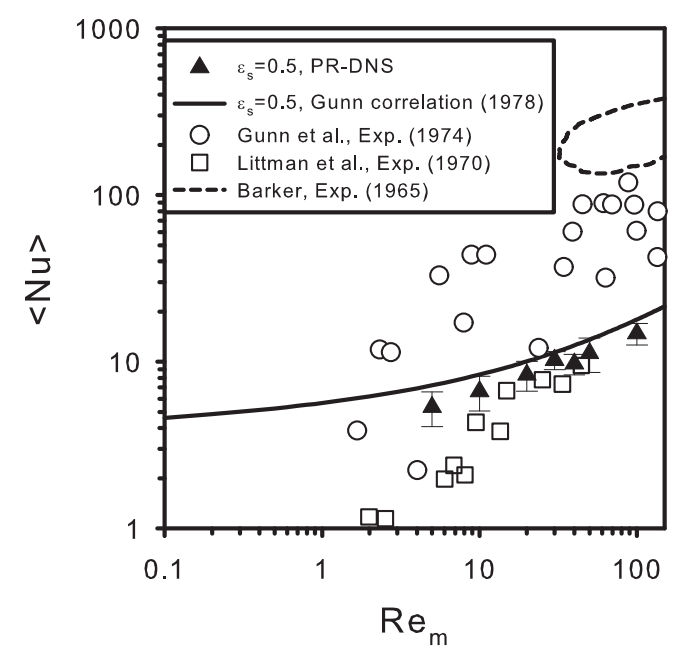

Figure 3: Comparison of Nusselt number from experimental data in packed beds with Gunn's correlation and PR-DNS data at $\varepsilon_{s}=0.5$. The triangles represent PR-DNS data with $95 \%$ confidence and the solid line represents Gunn's correlation (1978) at $\varepsilon_{s}=0.5$ that is close to a packed bed. The open circles represent the experimental data from Gunn et al. [21], the open squares represent experimental data from Littman et al. [53], and the range inside dashed line represents Barker's experimental data [54] in a packed bed.

\section{Nusselt number corresponding to average gas-solid heat transfer}

We now compare our PR-DNS data with Gunn's Nusselt number correlation [1] for gas-solid heat transfer that is widely used in two-fluid CFD models. We propose a new Nusselt number correlation for gas-solid flow that more closely matches our PR-DNS data and that also captures the Reynolds number dependence more accurately. The Nusselt number data obtained from our PUReIBM simulations are also compared with another gas-solid heat transfer PR-DNS reported by Tavassoli et al. [37]. We also compute the average gas-solid heat transfer in the two-fluid model using the Nusselt number from PR-DNS and compare it with the average gas-solid heat transfer extracted directly from PR-DNS. We find discrepancies that we are able to explain and reduce by proposing an improved model for average gas-solid heat transfer that is based on our model for the average bulk fluid temperature.

\subsection{Computation of Nusselt number from PR-DNS}

For gas-solid heat transfer, Gunn's Nusselt number correlation [1] of experimental data is used to compute the average gas-solid heat transfer in Eq. 28. 


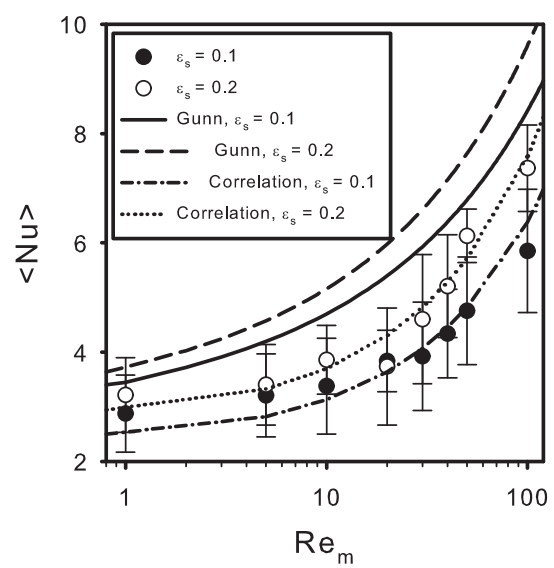

(a)

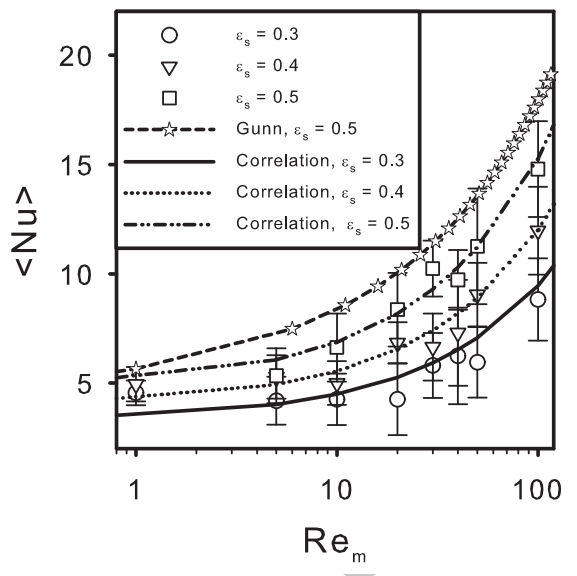

(b)

Figure 4: Dependence of the average Nusselt number in random particle assemblies on mean slip Reynolds number $\operatorname{Re}_{\mathrm{m}}$ for (a) low solid volume fraction $\left(\varepsilon_{s} \leq 0.2\right)$, and (b) higher solid volume fraction $\left(\varepsilon_{s} \geq 0.3\right)$ for gas-solid flow with $\operatorname{Pr}=0.7$. The symbols are the average Nusselt number from PR-DNS data and error bars indicate $95 \%$ confidence intervals using 5 MIS. The lines with symbols represent Gunn's correlation [1]. The lines without symbols represent the new PUReIBM Nusselt number correlation in Eq. 27.

Gunn's correlation [1] is written as the following function of Reynolds number Re, bed porosity $\varepsilon_{b}$, and Prandtl number Pr:

$$
\mathrm{Nu}=\left(7-10 \varepsilon_{b}+5 \varepsilon_{b}^{2}\right)\left(1+0.7 \operatorname{Re}^{0.2} \operatorname{Pr}^{1 / 3}\right)+\left(1.33-2.4 \varepsilon_{b}+1.2 \varepsilon_{b}^{2}\right) \operatorname{Re}^{0.7} \operatorname{Pr}^{1 / 3} .
$$

This correlation is valid in the bed porosity range $0.35 \leq \varepsilon_{b}=1-\varepsilon_{s} \leq 1.0$ and $1 \leq \operatorname{Re} \leq 10^{5}$. We extend Tenneti et al.'s [39] comparison of Nusselt number obtained from PR-DNS with Gunn's correlation to comprehensively compare Nusselt number over the range of Reynolds number $\operatorname{Re}_{m}$ and volume fraction $\varepsilon_{s}$ given in Table 1. Figure 4 shows the dependence of average Nusselt number on Reynolds number at low and high solid volume fractions.

The lines in Fig. 4(a) (line with symbols in Fig. 4(b)) are the Nusselt number obtained from Gunn's correlation [1] that is a fit to a collection of experimental data obtained from many sources. As expected, at a given volume fraction the average Nusselt number increases with Reynolds number due to an increase in the magnitude of the convection term. While the overall trend of the Nusselt number dependence on Reynolds number is captured by Gunn's correlation, it predicts a higher value of Nusselt number with Reynolds number than seen in 
the PR-DNS data. Gunn's correlation is within $20 \%$ of the PR-DNS data, and there are slight differences in the dependence on Reynolds number.

In order to place this comparison in context it should be noted that Gunn's correlation is an inspired fit to experimental data that vary by several orders of magnitude (see Fig. 3). On this scale, the difference between PR-DNS and Gunn's correlation of $20 \%$ is infinitesimal in comparison to the discrepancy between the correlation and the measurements themselves. An estimate of the contribution of free convection shows that it contributes less than $13 \%$ of the average Nusselt number even in the cases where it is expected to be important (low Reynolds number). The differences between the PR-DNS data and the experiment are more likely to arise from (i) experimental uncertainties (note the wide variation among the experimental studies themselves), (ii) assumption of constant gas properties in the simulations, (iii) the model assumption for axial conduction that is used to interpret the Nusselt number in the experimental reports, and (iv) at higher Reynolds number, the neglect of transport of temperature-velocity covariance in the 1-D model used to infer the Nusselt number in the experiments.

Figure 5 shows the dependence of the average Nusselt number on solid volume fraction for different Reynolds numbers. At a given Reynolds number, as the solid volume fraction increases so does the average Nusselt number. The dependence on solid volume fraction is explained on the basis that as the isothermal particle surfaces come closer, the temperature gradient in the fluid phase between them increases. In Fig. 5 we again see a systematic difference between Gunn's correlation and the PR-DNS data at a solid volume fraction of 0.5.

\subsection{Nusselt number correlation from $P R-D N S$}

In order to provide a correlation that better fits the PR-DNS data and to capture its dependence on Reynolds number more accurately, the following Nusselt number correlation for gas-solid flow is proposed by fitting our PR-DNS data:

$$
\mathrm{Nu}=\left(-0.46+1.77 \varepsilon_{b}+0.69 \varepsilon_{b}^{2}\right) / \varepsilon_{b}^{3}+\left(1.37-2.4 \varepsilon_{b}+1.2 \varepsilon_{b}^{2}\right) \operatorname{Re}^{0.7} \operatorname{Pr}^{1 / 3} .
$$

For simplicity of notation we drop the angle bracket notation in the expression for this correlation of average Nusselt number. This correlation is valid in the bed porosity range $0.5 \leq \varepsilon_{b} \leq 1.0$ and $1 \leq \mathrm{Re} \leq 100$. The first term in the new correlation accounts for the dependence of the Nusselt number on volume fraction. It is developed using the same $\varepsilon_{b}^{3}$ dependence as Tenneti et al. [30] 


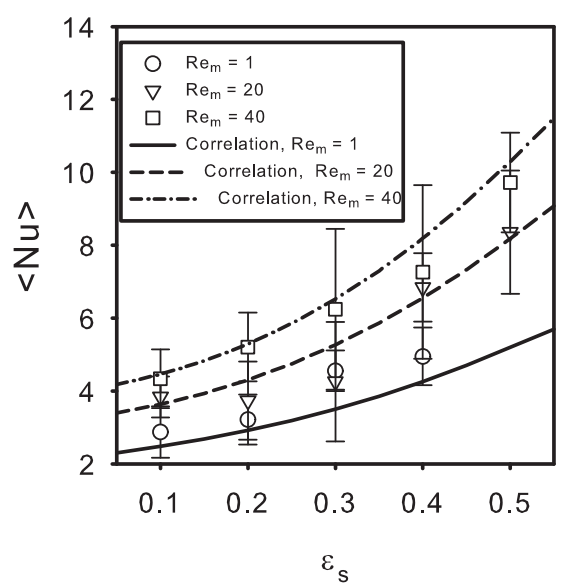

(a)

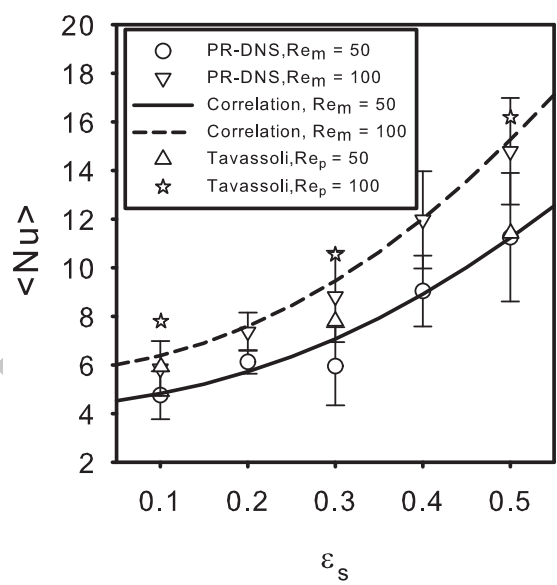

(b)

Figure 5: Dependence of the average Nusselt number in random particle assembly on solid volume fraction $\varepsilon_{s}$ (a) at low Reynolds number: $\operatorname{Re}_{\mathrm{m}}=1,20$ and 40, and (b) higher Reynolds number: $\operatorname{Re}_{\mathrm{m}}=50$ and 100, for gas-solid flow with $\operatorname{Pr}=0.7$. The symbols (open circle, open square, and downward triangle) are the average Nusselt number from PR-DNS data and error bars indicate $95 \%$ confidence intervals using 5 MIS. The other symbols (upward triangle and star) at $\varepsilon_{s}=0.1,0.3$, and 0.5 are the data from Tavassoli et al. [37]. The lines represent the PR-DNS Nusselt number correlation. 
employed to propose their PUReIBM drag correlation. This confirms in a limited sense the existence of a Reynolds analogy between drag and heat transfer in gas-solid flows. The second part of the PUReIBM correlation is the same as Gunn's correlation (see Eq. 26), and it completely represents the dependence of Nusselt number on Reynolds number. Note that in Gunn's correlation the first term also has a dependence on Reynolds number. As the Reynolds number and solid volume fraction tend to zero $\left(\varepsilon_{b} \rightarrow 1\right)$, this new PUReIBM correlation also yields the limiting value for the Nusselt number of 2, corresponding to conduction from a sphere.

In Fig. 5 the lines without symbols represent the PUReIBM Nusselt number correlation from PR-DNS data. The average difference between PR-DNS data and the PUReIBM correlation is about $7 \%$ for the range of Reynolds numbers considered. Fig. 5 also shows that the PUReIBM correlation closely fits most of the PR-DNS data except at low Reynolds number $\mathrm{Re}_{\mathrm{m}}=1$.

Figure 5(b) shows that our PR-DNS data compare with the average Nusselt number from Tavassoli et al. [37]. They also reported that the differences between their selected PR-DNS results and Gunn's correlation are about $20 \%$. Their average Nusselt number values are always larger than those from our PRDNS data. One possible explanation for this difference could be the difference in the setup of the gas-solid heat transfer problem in the two studies. In the Tavassoli et al. [37] study the flow domain includes both a thermally developing entrance region and a thermally fully-developed region, whereas in our setup there is only a thermal fully-developed region. Therefore, it is possible that Tavassoli et al. [37] obtained a higher value of the heat transfer coefficient (and Nusselt number) since the local heat transfer coefficient in the thermally developing entrance region for gas-solid flow is much higher than that in the thermal fully-developed region.

An important point to note is that the Nusselt number in Gunn's correlation as well as in our PR-DNS is computed in terms of the difference between the bulk fluid temperature (as shown in Fig. 6(a)) and the particle temperature. However, the two-fluid model (cf. Eq. 1) calculates the average gas-solid heat transfer in terms of the difference between the average fluid temperature (as shown in Fig. 6(b)) and the particle temperature. In Fig. 6(a), the average non-dimensional bulk fluid temperature from PR-DNS data denoted by open symbols decays rapidly due to fluid cooling in the streamwise direction. The same trend is also observed in the average non-dimensional fluid temperature as shown in Fig. 6(b). However, since the average non-dimensional bulk fluid 


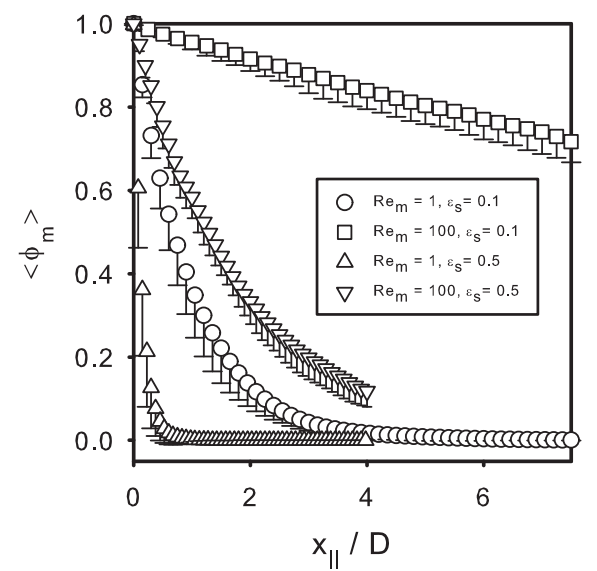

(a)

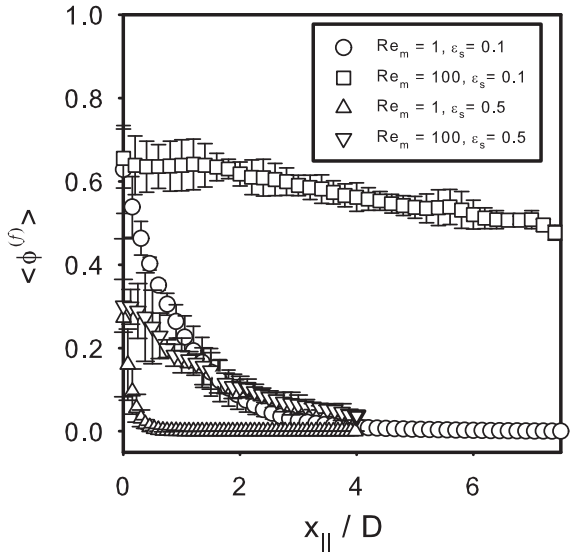

(b)

Figure 6: Axial variation of average non-dimensional bulk fluid temperature (Eq. 10) and cross-sectional average non-dimensional fluid temperature (Eq. 17) from PR-DNS data for $\varepsilon_{s}=0.1$ and 0.5 at mean slip Reynolds number of 1 and 100 . Error bars in both panels (shown only below the symbols in (a) for clarity) represent $95 \%$ confidence intervals inferred from 5 MIS.

includes the effect of the local velocity (cf. Eq. 6), we show in the following that it is inconsistent to use the Nusselt number defined in terms of the difference between the bulk fluid temperature and the particle temperature in the two-fluid model for average gas-solid heat transfer. Computational results quantify the magnitude of discrepancy arising from this inconsistency, and we then propose an improved model that reduces the discrepancy.

\section{Improved two-fluid model for average gas-solid heat transfer}

A widely used two-fluid model [40] for the average gas-solid heat transfer rate $\left\langle q_{j} \partial I_{f} / \partial x_{j}\right\rangle$ (cf. Eq. 1) is written in terms of the difference between average fluid temperature $\left\langle T^{(f)}\right\rangle$ and average solid temperature $\left\langle T^{(s)}\right\rangle$ as

$$
q_{T F}^{\prime \prime \prime}=\frac{6 k_{f} \varepsilon_{s} \mathrm{Nu}_{\mathrm{m}}}{D^{2}}\left(\left\langle T^{(s)}\right\rangle-\left\langle T^{(f)}\right\rangle\right),
$$

where $\varepsilon_{s}$ is the solid volume fraction, and $\mathrm{Nu}_{\mathrm{m}}$ is a model for the Nusselt number that is usually taken from a correlation to experimental data. This expression for the average volumetric gas-solid heat transfer rate $q_{T F}^{\prime \prime \prime}$ is valid for steady heat transfer in a homogeneous assembly of fixed monodisperse spherical particles, and is derived in Appendix B. 


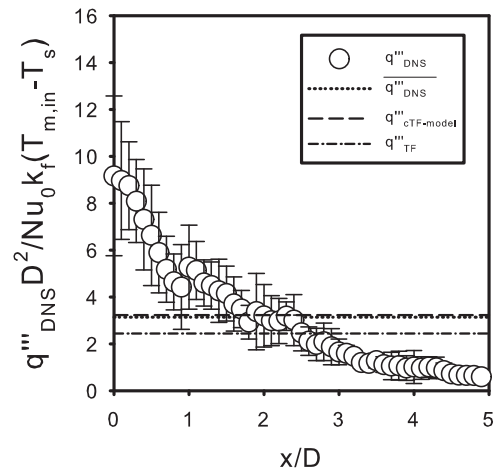

Figure 7: Variation of the normalized average volumetric heat transfer rate $q_{D N S}^{\prime \prime \prime}$ (cf. Eq. 58) with axial location $x_{\|}$at $\operatorname{Re}_{\mathrm{m}}=50$ and $\varepsilon_{s}=0.4$. The symbols represent the normalized average volumetric heat transfer rate $q_{D N S}^{\prime \prime \prime}$ and error bars indicate $95 \%$ confidence intervals using 5 MIS. $q_{D N S}^{\prime \prime \prime}$ is normalized by $h_{0}\left(T_{m, i n}-T_{s}\right) / D$, where $h_{0}$ is the heat transfer coefficient from the limiting value of Nusselt number $\mathrm{Nu}_{0}=h_{0} D / k_{f}=2\left(\right.$ at $\operatorname{Re}_{\mathrm{m}} \rightarrow 0$ and $\varepsilon_{s} \rightarrow 0$ ). The dotted line, dashed line, and dot-dashed line represent $\overline{q_{D N S}^{\prime \prime \prime}}$ in Eq. 58, $q_{C T F-\text { model }}^{\prime \prime \prime}$ in Eq. 54, and $q_{T F}^{\prime \prime \prime}$ in Eq. 57, respectively.

In order to verify the performance of this two-fluid model for average gassolid heat transfer, we compute the average volumetric interphase heat transfer rate $q_{T F}^{\prime \prime \prime}$ from the two-fluid model as

$$
q_{T F}^{\prime \prime \prime}=\frac{6 \varepsilon_{s} k_{f} \mathrm{Nu}}{D^{2}}\left(\left\langle T^{(s)}\right\rangle-\overline{\left\langle T^{(f)}\right\rangle}\right)
$$

(see Eq. 57 in Appendix C) where the PR-DNS data for average Nusselt number in Figs. 4 and 5 are used. Note that since $\left\langle T^{(f)}\right\rangle$ varies significantly along $x_{\|}$in many cases, the two-fluid definition of $\left\langle T^{(f)}\right\rangle$ in Eq. 28 is appropriately modified as

$$
\overline{\left\langle T^{(f)}\right\rangle}=\frac{1}{L} \int_{0}^{L}\left\langle T^{(f)}\right\rangle\left(x_{\|}\right) d x_{\|}
$$

(see Eq. 56 in Appendix C) when it is used in the two-fluid model.

We also directly compute the average volumetric interphase heat transfer rate $\overline{q_{D N S}^{\prime \prime \prime}}$ from PR-DNS data that is a statistical estimate of $\left\langle q_{\phi}^{\prime \prime \prime}\right\rangle$ (see Eq. 58 in Appendix C). Figure 7 shows a comparison of the normalized average volumetric interphase heat transfer rate from the two-fluid model and PR-DNS at $\operatorname{Re}_{\mathrm{m}}=50$ and $\varepsilon_{s}=0.4$. The magnitude of the normalized average volumetric interphase heat transfer rate $q_{D N S}^{\prime \prime \prime}$ has a large drop from 9.1 to 0.65 over the length of the particle bed. This decay of $q_{D N S}^{\prime \prime \prime}$ with axial location $x_{\|}$indicates 
that the gas-solid heat transfer is not homogeneous over $L=5 D$. The normalized difference between $\overline{q_{D N S}^{\prime \prime \prime}}$ and $q_{T F}^{\prime \prime \prime}$ is about $19 \%$. Fig. 9(a) shows that the normalized difference between $\overline{q_{D N S}^{\prime \prime \prime}}$ and $q_{T F}^{\prime \prime \prime}$ ranges from $5 \%$ to $36 \%$ over a range of Reynolds number and solid volume fraction, and increases with increasing solid volume fraction, with the maximum value occurring at $\varepsilon_{s}=0.5$. The average volumetric interphase heat transfer rate predicted by the two-fluid model $q_{T F}^{\prime \prime \prime}$ is considerably different from $\overline{q_{D N S}^{\prime \prime \prime}}$, even though the Nusselt number is taken from PR-DNS data. One reason for this difference is because of the inconsistency in using a Nusselt number based on the difference between bulk fluid temperature and the particle surface temperature in conjunction with a temperature difference based on the average fluid temperature in Eq. 28 to obtain the two-fluid model for the average volumetric interphase heat transfer rate $q_{T F}^{\prime \prime \prime}$. As noted earlier, the average Nusselt number in a fixed assembly of particles from PR-DNS is obtained in terms of the difference between the bulk fluid temperature and solid temperature as Eq. 39, and not the difference between the average fluid temperature and solid temperature. Thus, the difference between the two average volumetric interphase heat transfer rates results from using the average fluid temperature in the two-fluid model. Using a temperature difference based on the bulk fluid temperature is the correct way to compute the average volumetric interphase heat transfer rate in the two-fluid model, but the bulk fluid temperature is not solved as a field variable in the two-fluid model.

Our approach to remedy this problem is to express the average fluid temperature in the two-fluid model expression (Eq. 28) in terms of the bulk fluid temperature. In Appendix C, we use the relation between the average bulk fluid temperature and cross-sectional average fluid temperature in Eq. 17 to propose the following consistent two-fluid model for the average volumetric interphase heat transfer rate:

$$
q_{c T F-\text { model }}^{\prime \prime \prime}=\frac{6 \pi \varepsilon_{s} k_{f} \mathrm{Nu}}{4 D^{2}\left\langle\theta^{(f)}\right\rangle}\left(\left\langle T^{(s)}\right\rangle-\left\langle T^{(f)}\right\rangle\right),
$$

where the average scaled fluid temperature $\left\langle\theta^{(f)}\right\rangle$ can be extracted from PRDNS data. In fact, since the scaled fluid temperature $\theta$ is statistically homogeneous we can legitimately use volume averaging and subsequent ensembleaveraging over different particle configurations to compute $\left\langle\theta^{(f)}\right\rangle$ as $\left\langle\theta^{(f)}\right\rangle \cong$ $1 / M \sum_{\omega=1}^{M}\left\{\theta^{(f)}\right\}(\omega)$, where $\left\{\theta^{(f)}\right\}(\omega)=\int_{V_{f}} \theta(\mathbf{x} ; \omega) d V / V_{f}$. Figure 8 shows the variation of the average scaled fluid temperature $\left\langle\theta^{(f)}\right\rangle$ with Reynolds num- 


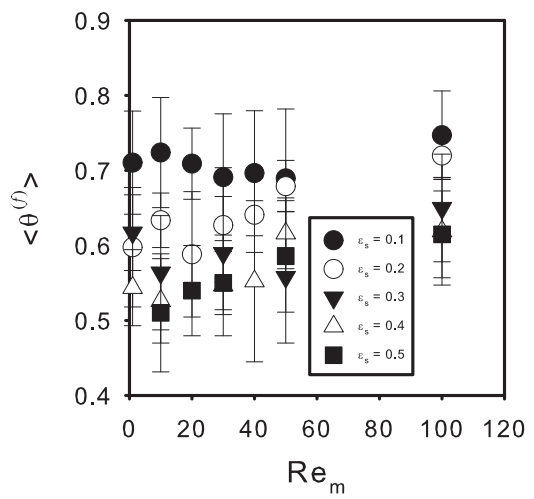

Figure 8: Variation of the average scaled fluid temperature $\left\langle\theta^{(f)}\right\rangle$ over Reynolds number of $1-100$ and volume fraction of $0.1-0.5$. Error bars represent $95 \%$ confidence intervals inferred from 5 MIS.

ber and volume fraction. For a given Reynolds number, the average scaled fluid temperature increases with increasing volume fraction. We also observe that the average scaled fluid temperature is only weakly dependent on Reynolds number. Based on this PR-DNS data we propose the following correlation for the average scaled fluid temperature

$$
\left\langle\theta^{(f)}\right\rangle=1-1.6 \varepsilon_{s}\left(1-\varepsilon_{s}\right)-3 \varepsilon_{s}\left(1-\varepsilon_{s}\right)^{4} \exp \left(-\operatorname{Re}_{\mathrm{m}}^{0.4} \varepsilon_{s}\right)
$$

that fits PR-DNS data with an average error of $5 \%$. This correlation is a function of solid volume fraction and Reynolds number, and can be used to relate the mean fluid temperature to the bulk fluid temperature. In the limiting case of infinite dilution (i.e. solid volume fraction $\varepsilon_{s} \rightarrow 0$ ), the average scaled fluid temperature $\left\langle\theta^{(f)}\right\rangle$ is equal to one. This limiting value is consistent with the fact that for flow without particles the temperature field is uniform.

Using the expressions for average volumetric interphase heat transfer rate in Appendix C, Fig. 7 shows that the difference between $q_{c T F-\text { model }}^{\prime \prime \prime}$ (see Eq. 54) and $\overline{q_{D N S}^{\prime \prime \prime}}$ (see Eq. 58) is about $8 \%$ and less than the difference between $q_{T F}^{\prime \prime \prime}$ and $\overline{q_{D N S}^{\prime \prime \prime}}$ at a solid volume fraction of 0.4. Thus, although $q_{D N S}^{\prime \prime \prime}$ is inhomogeneous along the axial location, $q_{c T F-\text { model }}^{\prime \prime \prime}$ is still close to the PR-DNS data. Figure 9(b) compares the average volumetric interphase heat transfer rate from this consistent two-fluid model and PR-DNS data over a wide range of Reynolds number and solid volume fraction. The maximum value of the difference between $\overline{q_{D N S}^{\prime \prime \prime}}$ and $q_{c T F-\text { model }}^{\prime \prime \prime}$ is about $12 \%$. For the cases where scale separation 
holds $\left(\varepsilon_{s}=0.1, \operatorname{Re}_{m} \geq 20\right.$ ) we find that the difference is very low (the maximum of the difference is about $7 \%$ ). Therefore, for the scale separated cases the consistent two-fluid model that uses the bulk fluid temperature difference is very accurate. Thus, the consistent model that uses the bulk fluid temperature difference with the Nusselt number correlation based on the bulk fluid temperature results in significant improvement of the predicted average gas-solid heat transfer rate.

For the rest of the cases the error most probably arises from the spatial inhomogeneity of the average fluid temperature in the domain (cf. Eq. 56 and Fig. 7). Even so, for most of the cases the difference between $\overline{q_{D N S}^{\prime \prime \prime}}$ and $q_{c T F-\text { model }}^{\prime \prime \prime}$ is below $12 \%$, whereas as noted earlier the difference between $\overline{q_{D N S}^{\prime \prime \prime}}$ and $q_{T F}^{\prime \prime \prime}$ (see Fig. 9(a)) are in the range of 5-36\%. However, these errors should be interpreted cautiously since they relied on having the spatial variation of the average fluid temperature from the PR-DNS, which would not be the case in practical application of the two-fluid model. Nevertheless, this improved consistent two-fluid model (see Eq. 29) for the average volumetric interphase heat transfer rate that uses the average scaled fluid temperature $\left\langle\theta^{(f)}\right\rangle$ correlation (see Eq. 30) can be directly used in existing multiphase CFD codes that are based on the two-fluid model.

\section{Discussion}

The results for gas-solid heat transfer presented here have been obtained under certain simplifying assumptions. Here we discuss the applicability of the results and identify areas for future work that would extend these results. As noted earlier, the principal assumptions in this work are: (i) isothermal particles with a single spatially uniform temperature for all particles that is constant in time, and (ii) neglect of radiation and free convection. Clearly the Nusselt number correlation can depend on the temperature boundary condition at the particle surface. A more faithful description would involve a coupled solution of the conduction problem inside each sphere. Such computations would certainly be more computationally expensive but are not out of reach for future studies. An intermediate simpler approach would be to assume a uniform temperature inside each solid particle (infinite conductance limit that can be characterized by the Biot number) and yet allow each particle's temperature to vary in time. Table 2 shows the Biot number for typical particles encountered in the applications that motivate this study. In each of these applications it seems that a 


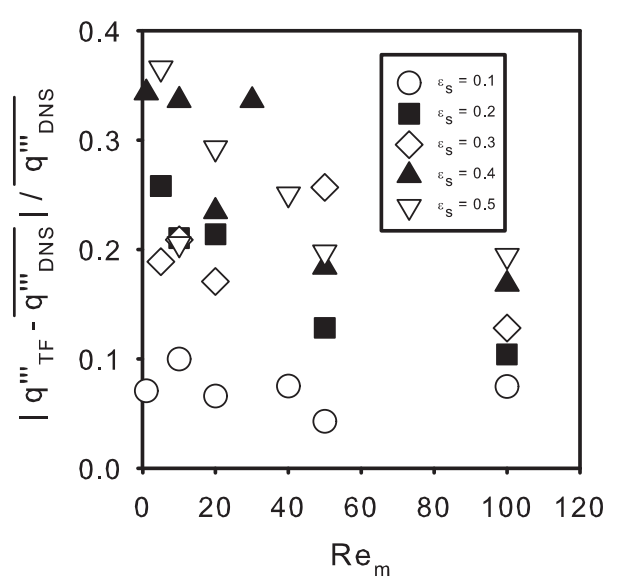

(a)

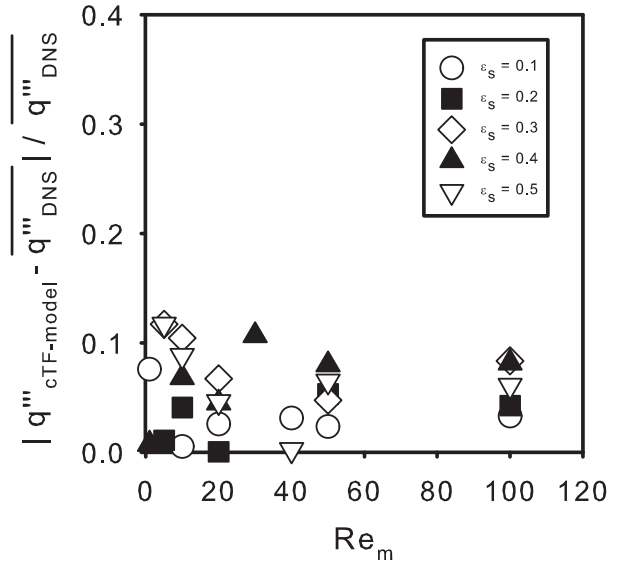

(b)

Figure 9: Comparison of average volumetric interphase heat transfer rate over a range of solid volume fraction and mean slip Reynolds number with $\overline{q_{D N S}^{\prime \prime \prime}}$ (see Eq. 58) from PR-DNS data: (a) the standard two-fluid model (see Eq. 57), and (b) the newly improved consistent twofluid (cTF) model (see Eq. 54). The average Nusselt number and average fluid temperature in both models are taken from PR-DNS.

uniform particle temperature is a reasonable assumption for small particle diameters, and only in the biomass application with large particle diameters is spatial temperature inhomogeneity inside the particle likely to be important. Smaller particle diameters also more closely satisfy the neglect of free convection, as discussed in detail in Tenneti et al.'s paper [39].

It is also useful to estimate the validity of the assumption of a constant particle temperature that is not varying in time. Table 2 also shows the ratio of the particle thermal response time to the mean flow through time over a particle diameter. This shows that the particle thermal response time is usually much larger than the flow through time with the ratio ranging from 10 to 3895 . The typical box size in the PR-DNS simulations is at most $8 D$ and therefore only in the case of biomass particles do we expect the particle temperature to change on a time scale comparable to the transit time for the flow through the domain. In future studies it would be relatively simple to use a lumped capacitance model and allow particle temperatures to vary in time.

Since the Nusselt number correlation inferred from PR-DNS in this study is obtained using data from heat transfer in fixed beds (in fact, so is Gunn's correlation), care should be taken when applying it to fluidized beds. The results 


\begin{tabular}{|c|c|c|c|c|c|c|c|c|c|c|}
\hline & $\begin{array}{c}D \\
(\mu \mathrm{m})\end{array}$ & $\begin{array}{c}\rho_{s} \\
\left(\mathrm{~kg} / \mathrm{m}^{3}\right)\end{array}$ & $\begin{array}{c}c_{p, s} \\
(\mathrm{~kJ} / \mathrm{kg} \cdot \mathrm{K})\end{array}$ & $\begin{array}{c}\rho_{f} \\
\left(\mathrm{~kg} / \mathrm{m}^{3}\right)\end{array}$ & $\begin{array}{c}c_{p, f} \\
(\mathrm{~kJ} / \mathrm{kg} \cdot \mathrm{K})\end{array}$ & $\begin{array}{c}\rho_{s} c_{p, s} / \\
\rho_{f} c_{p, f}\end{array}$ & $\begin{array}{c}k_{s} \\
(\mathrm{~W} / \mathrm{m} \cdot \mathrm{K})\end{array}$ & $\begin{array}{c}k_{f} \\
(\mathrm{~W} / \mathrm{m} \cdot \mathrm{K})\end{array}$ & $\mathrm{Bi}$ & $\begin{array}{c}\tau_{L c} / \\
\tau_{\text {flow }}\end{array}$ \\
\hline $\begin{array}{l}\mathrm{CO}_{2} \\
\text { cap- } \\
\text { ture }\end{array}$ & 401 & 2394 & 3.93 & 1.77 & 0.85 & 6254 & 0.5 & 0.017 & $\begin{array}{c}0.06- \\
0.7\end{array}$ & $\begin{array}{c}36- \\
1897\end{array}$ \\
\hline CLC & 100 & 2960 & 2.1 & 0.44 & 1.1 & 12843 & 0.9 & 0.052 & $\begin{array}{c}0.11- \\
1.2\end{array}$ & $\begin{array}{c}74- \\
3895\end{array}$ \\
\hline Biomass & 500 & 400 & 2.27 & 0.42 & 1.22 & 1772 & 0.6 & 0.054 & $\begin{array}{c}0.18- \\
1.8\end{array}$ & $10-537$ \\
\hline
\end{tabular}

Table 2: Typical particle properties and non-dimensional parameters such as the Biot number and the time scale ratio $\tau_{L c} / \tau_{\text {flow }}$ that are encountered in gas-solid heat transfer applications such as $\mathrm{CO}_{2}$ capture [5], chemical looping combustion (CLC) [3], and biomass pyrolysis [55, 56]. In $\mathrm{CO}_{2}$ capture the gas phase is $\mathrm{CO}_{2}$ and the solid phase is $\mathrm{NaCO}_{3}$; in $\mathrm{CLC}$ the gas phase is $\mathrm{CO}$ and the solid phase is $\mathrm{CaSO}_{4}$; in biomass pyrolyisis the gas phase is $N_{2}$ and the solid phase is bagasse. The Biot number is defined as $\mathrm{Bi}=h D / k_{s}$, where the heat transfer coefficent is computed by $h=\mathrm{Nu} k_{f} / D$, and the Nusselt number is in the range $2--20$ from our simulations. The particle thermal response time for a sphere is defined as $\tau_{L c}=\rho_{s} c_{p, s} V / A_{s} h=\rho_{s} c_{p, s} D / 6 h$, and the flow through time is $\tau_{\text {flow }}=D /|\langle\mathbf{W}\rangle|$.

from this study show that the mean fluid temperature can vary significantly over a few particle diameters, and it might be expected that particle motion in this mean temperature gradient can result in a change in the Nusselt number. Note that in the hydrodynamic problem there is no mean velocity gradient because the velocity field is homogeneous. Therefore, in the hydrodynamic problem the drag force and pseudo-turbulent kinetic energy for high Stokes number particles in freely evolving suspension are well approximated by fixed-bed computations (see Tenneti [45] and Mehrabadi et al. [57]). Heat transfer in freely evolving suspension of gas-solid flow should be performed for a complete verification but these are outside the scope of this study.

Although simulating a thermally fully developed flow allows us to propose Nusselt number correlations with relatively low statistical variability, this study does not account for entrance length effects. Now that we have the thermally fully developed results in hand, we plan to investigate the effect of entrance length on the heat transfer problem by using inflow/outflow boundary conditions in future reports.

The Nusselt number correlation proposed in this work is restricted to gassolid flow. Simulation of heat transfer in liquid-solid flow requires considerably higher grid resolution to resolve the thermal boundary layers that correspond to high Prandtl number. It is of interest to consider whether the Nusselt number correlation proposed in this work can also be used to compute the Sherwood 
number in a mass transfer problem on the basis of the Reynolds analogy. However, if mass transfer is strongly coupled to heat transfer, as is often the case in gas-solid flows, then the Sherwood number will depend not only on the Reynolds number and Schmidt number, but also on the Lewis number. The validity of the Reynolds heat/mass transfer analogy in gas-solid flows is a worthwhile topic for future study. Finally, it should be noted that these results are applicable to steady heat transfer and they do not account for the effect of unsteady mean temperature effects on the Nusselt number in gas-solid flow.

\section{Conclusions}

PR-DNS simulations of gas-solid heat transfer in steady flow through a homogeneous fixed assembly of particles is used to verify the assumptions underlying the continuum formulation of averaged equations, and to quantify the average gas-solid heat transfer term in the average fluid temperature equation over a range of mean slip Reynolds numbers (1-100) and volume fractions (0.1$0.5)$. PR-DNS data for the Nusselt number as a function of Reynolds number $\operatorname{Re}_{\mathrm{m}}$ and volume fraction $\varepsilon_{s}$ is compared with Gunn's correlation [1]. The average Nusselt number computed from PR-DNS data is close to Gunn's correlation with an average difference of $20 \%$. A new PUReIBM Nusselt number correlation is proposed using PR-DNS data with an average difference of $7 \%$ that captures the Reynolds number dependence more accurately. This correlation is valid in a range of $0.5 \leq \varepsilon_{b} \leq 1.0$ and $1 \leq \mathrm{Re} \leq 100$.

The average gas-solid heat transfer in the two-fluid model is computed based on the average Nusselt number and the difference between average particle temperature and average fluid temperature, which can result in $5 \%$ to $36 \%$ difference when compared with the average gas-solid heat transfer obtained directly from PR-DNS data. These results indicate that rather than the average fluid temperature, it is the bulk fluid temperature that is the correct choice to compute average gas-solid heat transfer in gas-solid flow. Using a relation between the average bulk fluid temperature and average fluid temperature, an improved model for the average gas-solid heat transfer is developed in terms of average scaled fluid temperature $\left\langle\theta^{(f)}\right\rangle$, average Nusselt number $\langle\mathrm{Nu}\rangle$, volume fraction $\varepsilon_{s}$, and the difference between the average fluid temperature $\left\langle T^{(f)}\right\rangle$ and average solid temperature $\left\langle T^{(s)}\right\rangle$. This model can be directly used for computing the average gas-solid heat transfer in two-fluid computations. 

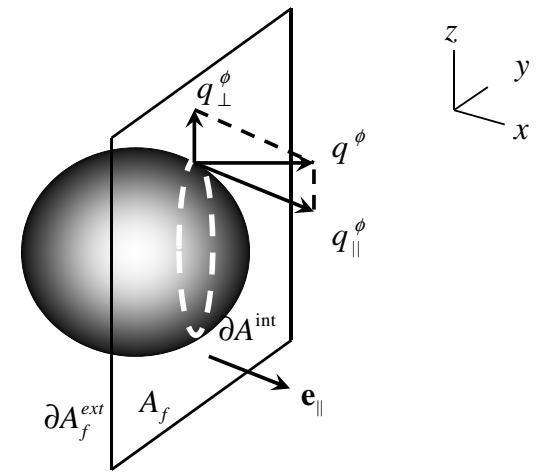

Figure 10: Sketch of physical domain with a particle intersecting the cross-sectional plane $(y-z$ plane $)$ normal to the streamwise direction. The cross-sectional area occupied by fluid is denoted $A_{f}$. The exterior boundary of the fluid phase in the plane is denoted $\partial A_{f}^{e x t}$. The boundary between the fluid phase and solid phase is denoted $\partial A^{\text {int }}$. The normal vector $\mathbf{e}_{\|}$ denotes the streamwise direction. $q_{||}^{\phi}$ and $q_{\perp}^{\phi}$ are the in-plane and out-of-plane heat fluxes, respectively.

\section{A. Computation of Nusselt number}

While in internal forced convection in a pipe flow [44] the heat flux vector at the pipe wall is perpendicular to the solid surface and always lies in the crosssectional plane for pipes of constant cross-section, in gas-solid heat transfer there exists a component of the local interphase heat flux vector along the streamwise direction. This is due to the fact that the interphase normal at the particle surface changes direction in gas-solid flow with changing axial location. For quantifying the unclosed terms using PR-DNS, it turns out to be convenient to distinguish between two components of the local interphase heat flux: (i) the component along the streamwise or axial direction, which is denoted the out-ofplane local interphase heat flux, and (ii) the component of the local interphase heat flux normal to the streamwise direction, or the in-plane local interphase heat flux (see Fig. 10). In order to quantify the local interphase heat flux, the PR-DNS instantaneous fluid temperature equation (Eq. 21) is integrated over $A_{f}$, which denotes the portion of the cross-sectional area that is occupied by fluid, in the $y-z$ plane perpendicular to the streamwise direction. For steady 
flow we obtain the following equation:

$$
\frac{\rho_{f} c_{p f}}{A} \int_{A_{f}} \frac{\partial\left(u_{j} \phi\right)}{\partial x_{j}} d A=\frac{1}{A} \int_{A_{f}}-\frac{\partial q_{j}^{\phi}}{\partial x_{j}} d A
$$

The divergence term on the right hand side of Eq. 31 is first expressed in terms of the out-of-plane and in-plane components of the heat flux vector $\mathbf{q}^{\phi}=q_{\|}^{\phi} \mathbf{e}_{\|}+$ $q_{\perp}^{\phi} \mathbf{e}_{\perp}$, and then the divergence theorem is used in the $y$ - $z$ plane for the in-plane component to obtain:

$$
\underbrace{\frac{1}{A} \int_{A_{f}} \frac{\partial q_{j}^{\phi}}{\partial x_{j}} d A}_{\text {RHS }}=\underbrace{\frac{1}{A} \int_{A_{f}} \frac{\partial q_{\|}^{\phi}}{\partial x_{\|}} d A}_{I}+\underbrace{\frac{1}{A} \oint_{\partial A_{f}^{e x t}} q_{j, \perp}^{\phi} \cdot n_{j, \perp}^{(e x t)} d l}_{I I}-\underbrace{\frac{1}{A} \oint_{\partial A^{i n t}} q_{j, \perp}^{\phi} \cdot n_{j, \perp}^{(s)} d l}_{I I I},
$$

where $l$ is the perimeter of circles formed by the intersection of particles in the cross-sectional plane, and $q_{j, \perp}^{\phi}$ is the in-plane interphase heat flux, and $n_{j, \perp}^{(s)}$ is the in-plane component of the outward unit normal vector on the surface of particles. Note that since the heat flux is defined in terms of the non-dimensional temperature $\phi$, its units are $\mathrm{W} /\left(\mathrm{m}^{2}-\mathrm{K}\right)$. Term I represents the streamwise gradient of out-of-plane heat flux in the cross-sectional plane. Term II represents the net conduction of heat flux into this plane from exterior boundaries of the fluid phase at the domain boundary, while Term III represents in-plane interphase heat transfer from particle to fluid. Term II is equal to zero due to periodic boundary conditions on the non-dimensional temperature field $\phi$ in the $y$ and $z$ directions. Term III is defined as the volumetric heat transfer rate per unit temperature difference corresponding to the in-plane local interphase heat flux

$$
q_{\perp}^{\prime \prime \prime}\left(x_{\|} ; \omega\right)=\frac{1}{A} \oint_{\partial A^{i n t}} q_{j, \perp}^{\phi} \cdot n_{j, \perp}^{(s)} d l,
$$

where the unit for $q_{\perp}^{\prime \prime \prime}\left(x_{\| \mid} ; \omega\right)$ is $\mathrm{W} /\left(\mathrm{m}^{3}-\mathrm{K}\right)$, and this quantity is specific to the realization $\omega$ that corresponds to a particular configuration of particles.

Term I can be decomposed into an axial conduction term and the axial (outof-plane) contribution to the interphase heat flux using the indicator function in the fluid phase $I_{f}$ as follows:

$$
\frac{1}{A} \int_{A_{f}} \frac{\partial q_{\|}^{\phi}}{\partial x_{\|}} d A=\frac{1}{A} \int_{A} I_{f} \frac{\partial q_{\|}^{\phi}}{\partial x_{\|}} d A=\frac{1}{A} \int_{A} \frac{\partial I_{f} q_{\|}^{\phi}}{\partial x_{\|}} d A+\frac{1}{A} \int_{A} q_{\|}^{\phi} \frac{\partial I_{f}}{\partial x_{\|}} d A
$$

The first term on the RHS of the above equation is the axial conduction in the 
fluid phase (this is quantified and modeled in Part 2 of this two-part series). We define the axial conduction in the fluid phase at axial location $x_{||}$for realization $\omega$ as

$$
q_{\text {cond }}^{\prime \prime \prime}\left(x_{\|} ; \omega\right)=\frac{1}{A} \int_{A} \frac{\partial I_{f} q_{\|}^{\phi}}{\partial x_{\|}} d A .
$$

The second term on the RHS of Eq. 34 is the volumetric heat transfer rate corresponding to the out-of-plane local interphase heat flux $q_{\|}^{\phi}$ :

$$
q_{\|}^{\prime \prime \prime}\left(x_{\|} ; \omega\right)=\frac{1}{A} \int_{A} q_{\|}^{\phi} \frac{\partial I_{f}}{\partial x_{\|}} d A .
$$

It is clearly seen that due to the presence of particles, Term I includes the axial conduction in the fluid phase and the out-of-plane local interphase heat flux. The latter does not appear in single-phase flow.

Combining the in-plane and out-of-plane local interphase heat flux, we define the local volumetric interphase heat transfer rate $q_{\phi}^{\prime \prime \prime}\left(x_{\|} ; \omega\right)$ at axial location $x_{\|}$ in realization $\omega$ as

$$
q_{\phi}^{\prime \prime \prime}\left(x_{\|} ; \omega\right)=q_{\| \mid}^{\prime \prime \prime}\left(x_{\|} ; \omega\right)+q_{\perp}^{\prime \prime \prime}\left(x_{\|} ; \omega\right) .
$$

In the cross-sectional plane at every axial location $x_{||}$we define the local convective heat transfer coefficient $h\left(x_{\|} ; \omega\right)$ corresponding to heat transfer between fluid and particles following Bird et al. [58]:

$$
A q_{\phi}^{\prime \prime \prime}\left(x_{\| \mid} ; \omega\right)=h\left(x_{\|} ; \omega\right) P\left(x_{\|} ; \omega\right) \phi_{m}\left(x_{\|} ; \omega\right),
$$

where $P\left(x_{\|} ; \omega\right)$ is the perimeter formed by cutting the particles in the crosssectional plane, $A$ is the cross-sectional area, and the non-dimensional bulk temperature $\phi_{m}\left(x_{\|} ; \omega\right)$. The left hand side term in Eq. 38 represents the heat transfer rate per unit length of interface in the cross-sectional plane and its units are $\mathrm{W} /(\mathrm{m}-\mathrm{K})$.

Based on the local convective heat transfer coefficient $h\left(x_{\|} ; \omega\right)$ at axial location $x_{\|}$a local Nusselt number can be defined. The local Nusselt number at axial location $x_{||}$for realization $\omega$ is:

$$
\mathrm{Nu}\left(x_{\|} ; \omega\right)=\frac{h\left(x_{\|} ; \omega\right) D}{k_{f}}=\frac{A q_{\phi}^{\prime \prime \prime}\left(x_{\|} ; \omega\right)}{k_{f} P\left(x_{\|} ; \omega\right) \phi_{m}\left(x_{\|} ; \omega\right)} D .
$$

The local Nusselt number can then used to calculate an average Nusselt number at axial location $x_{\|}$, where in this context we use the term average to 
mean an ensemble-average over different particle configurations:

$$
\left\langle\mathrm{Nu}\left(x_{\|}\right)\right\rangle_{M}=\frac{1}{M} \sum_{\omega=1}^{M} \mathrm{Nu}\left(x_{\|} ; \omega\right) .
$$

Similarly, the ensemble-average non-dimensional bulk fluid temperature $\left\langle\phi_{m}\left(x_{\|}\right)\right\rangle$ at each axial location can also be estimated using $M$ realizations as:

$$
\left\langle\phi_{m}\left(x_{||}\right)\right\rangle_{M}=\frac{1}{M} \sum_{\omega=1}^{M} \phi_{m}\left(x_{\|} ; \omega\right) .
$$

For the case of thermally fully developed flow past a homogeneous fixed particle assembly, the Nusselt number is homogeneous in the streamwise direction [39]. Therefore, the average Nusselt number $\langle\mathrm{Nu}\rangle$ can be estimated by integrating Eq. 40 over the axial length of the box:

$$
\langle\mathrm{Nu}\rangle \cong\{\mathrm{Nu}\}_{M, V}=\frac{1}{L} \int_{0}^{L}\left\langle\mathrm{Nu}\left(x_{\|}\right)\right\rangle_{M} d x_{\|},
$$

where $\{\mathrm{Nu}\}_{V, M}$ denotes an estimate to the expectation $\langle\mathrm{Nu}\rangle$.

In the context of establishing grid convergence with respect to a single configuration of particles for thermally fully developed flow past a fixed assembly of particles, it is useful to define a volume-averaged Nusselt number for that realization:

$$
\{\mathrm{Nu}\}_{V}(\omega)=\frac{1}{L} \int_{0}^{L} \mathrm{Nu}\left(x_{\|} ; \omega\right) d x_{\|}
$$

\section{B. Average gas-solid heat transfer in the two-fluid model}

We derive the expression for the average volumetric interphase heat transfer rate in the two-fluid model (Eq. 28) for monodisperse spherical particles. Consider a cubical control volume of side $L$ in which there is steady unidirectional flow in the $x_{\|}$direction past $N$ spherical particles each of diameter $D$, with average fluid temperature $\left\langle T^{(f)}\right\rangle$ and average solid temperature $\left\langle T^{(s)}\right\rangle$. The average volumetric interphase heat transfer rate $q_{T F}^{\prime \prime \prime}$ in the domain is given by

$$
V q_{T F}^{\prime \prime \prime}=A_{s} \bar{h}\left(\left\langle T^{(s)}\right\rangle-\left\langle T^{(f)}\right\rangle\right)
$$

where $A_{s}=N \pi D^{2}$ is the total surface area of particles, $V=L^{3}$ is the volume of this domain, and $\bar{h}$ is the average heat transfer coefficient. In the above equation, the ratio of particle surface area to domain volume can be written in 
terms of the of solid volume fraction $\varepsilon_{s}$ and particle diameter as:

$$
\frac{A_{s}}{V}=\frac{N \pi D^{2}}{L^{3}}=\frac{N \pi D^{3}}{6 L^{3}} \frac{6}{D}=\frac{6 \varepsilon_{s}}{D} .
$$

Based on the definition of the Nusselt number $\mathrm{Nu}_{\mathrm{m}}=\bar{h} D / k_{f}$, the average volumetric interphase heat transfer rate in the two-fluid model is obtained as

$$
q_{T F}^{\prime \prime \prime}=\frac{6 \varepsilon_{s} k_{f} \mathrm{Nu}_{\mathrm{m}}}{D^{2}}\left(\left\langle T^{(s)}\right\rangle-\left\langle T^{(f)}\right\rangle\right),
$$

with units $\mathrm{W} / \mathrm{m}^{3}$. In this expression the difference between average fluid temperature and average solid temperature is used to compute the average volumetric interphase heat transfer rate. Note that since the two-fluid model for the average volumetric interphase heat transfer rate $q_{T F}^{\prime \prime \prime}$ is defined in terms of temperature difference, its units are different from that of $q_{\phi}^{\prime \prime \prime}$. However, Bird et al. [58] uses the difference between bulk fluid temperature and average solid temperature to compute the average gas-solid heat transfer in a fixed bed of particles. In the following Appendix, we will show how an improved consistent two-fluid model to compute average volumetric interphase heat transfer is developed based on bulk fluid temperature.

\section{Improved model for average volumetric interphase heat transfer rate}

The expression for the average volumetric interphase heat transfer rate in the two-fluid model derived in Appendix B assumes local homogeneity of the average fluid and solid temperature fields. Here we derive an extension of that model that is applicable to a spatially inhomogeneous average fluid temperature field, such as encountered in the gas-solid heat transfer problem simulated by PR-DNS in this work.

We begin with Eq. 38 that relates the local volumetric interphase heat transfer rate $q_{\phi}^{\prime \prime \prime}\left(x_{\|} ; \omega\right)$ at an axial location $x_{||}$for realization $\omega$ with $\phi_{m}\left(x_{\|} ; \omega\right)$, which is the non-dimensional difference between the bulk fluid temperature and the particle surface temperature. Taking the ensemble-average of Eq. 38 results in

$$
\left\langle q_{\phi}^{\prime \prime \prime}\right\rangle\left(x_{\|}\right)=\langle h\rangle\left(x_{\|}\right) \frac{\langle P\rangle\left(x_{\|}\right)}{A}\left\langle\phi_{m}\right\rangle\left(x_{\|}\right),
$$

where $\left\langle q_{\phi}^{\prime \prime \prime}\right\rangle\left(x_{\|}\right)$(cf. Eq. 8) is the average volumetric interphase heat transfer rate per unit temperature difference. We define the inhomogeneous average heat 
transfer coefficient $\langle h\rangle\left(x_{\|}\right)$to be

$$
\langle h\rangle\left(x_{\|}\right) \equiv \frac{A\left\langle q_{\phi}^{\prime \prime \prime}\right\rangle\left(x_{\|}\right)}{\langle P\rangle\left(x_{\|}\right)\left\langle\phi_{m}\right\rangle\left(x_{\| \mid}\right)} .
$$

Note that in general the average of a product of random variables is not equal to the product of the averages, unless the random variables are uncorrelated. Here we are not assuming that the variables on the right hand side of Eq. 38 are uncorrelated, but we are assuming in the above expression that the dependence of $\phi_{m}\left(x_{\|} ; \omega\right)$ and $P\left(x_{\|} ; \omega\right)$ on the particle configuration can be expressed as a dependence on the average solid volume fraction $\varepsilon_{s}$, and any correlation of the three right hand side terms can be captured in the definition of the inhomogeneous average heat transfer coefficient $\langle h\rangle\left(x_{\|}\right)$in Eq. 48. It should however be noted that this model cannot capture the dependence of the inhomogeneous average heat transfer coefficient on clustered arrangements of homogeneous particle fields where the volumetric interphase heat transfer rate could depend on the pair correlation function of the particles.

Now although the average volumetric interphase heat transfer rate $\left\langle q_{\phi}^{\prime \prime \prime}\right\rangle\left(x_{\|}\right)$ and $\left\langle\phi_{m}\right\rangle\left(x_{||}\right)$are inhomogeneous in $x_{||}$for the gas-solid flow problem considered in this work, the particle configuration is statistically homogeneous. Therefore, the average perimeter $\langle P\rangle\left(x_{\|}\right)$does not depend on $x_{\|}$. An simple expression for the average perimeter $\langle P\rangle$ in terms of the average solid volume fraction is now derived.

We need to calculate the average perimeter corresponding to the intersection of the $y-z$ plane located at $x_{\|}$with a random assembly of monodisperse spheres as shown in Fig. 11. Since the particle field is statistically homogeneous, the $y-z$ plane intersects spheres at various axial locations, and the axial locations reckoned from their respective sphere centers are distributed with equal probability in $(-R, R)$, where $R$ is the sphere radius. In other words, if the axial coordinate of the sphere center is $X_{c}$ and the $y-z$ plane is located at $x_{\|}$, then $X=X_{c}-x_{\|}$ is a random variable uniformly distributed in $(-R, R)$. If the radius of the circle formed by the intersection of the $y-z$ plane with the sphere is $R_{\perp}$, then

$$
\langle P\rangle=\langle N\rangle \int_{+R}^{-R} 2 \pi R_{\perp} f_{X} d x,
$$

where $\langle N\rangle$ is the average number of spheres in the volume $A \times D$ ( $A$ being the cross-sectional area of the plane and $D$ being the sphere diameter), $f_{X}=1 / 2 R$, 


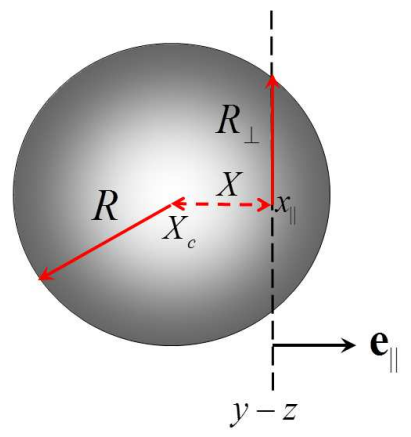

Figure 11: Sketch of computation of the average perimeter corresponding to the intersection of the $y-z$ plane located at $x_{\|}$. The sphere radius is $R$ and $R_{\perp}$ is the radius of the circle formed by the intersection of the $y-z$ plane with the sphere. The axial coordinate of the sphere center is $X_{c}$ and a random variable uniformly distributed in $(-R, R)$ is $X=X_{c}-x_{\|}$. The normal vector $\mathbf{e}_{\|}$denotes the streamwise direction.

and the integration limits correspond to the traversal of a sphere from just touching the plane with $X_{c}=x_{\|}-R$ to $X_{c}=x_{\|}+R$. Noting that $R_{\perp}=$ $R \sin \theta=\sqrt{R^{2}-X^{2}}$ the above integral can be simplified to yield

$$
\langle P\rangle=2 \pi\langle N\rangle R \frac{\pi}{4}=\frac{\pi^{2}\langle N\rangle D}{4} .
$$

Substituting $\langle N\rangle=n A D$, where $n$ is the number density that is related to the average solid volume fraction by $\varepsilon_{s}=n \pi D^{3} / 6$, results in the following expression for $\langle P\rangle / A$ :

$$
\frac{\langle P\rangle}{A}=\frac{6 \pi \varepsilon_{s}}{4 D},
$$

which is close to the geometrical factor in the original two-fluid model. This leads to the final expression for the inhomogeneous average volumetric heat transfer rate

$$
\left\langle q_{\phi}^{\prime \prime \prime}\right\rangle\left(x_{||}\right)=\langle h\rangle\left(x_{||}\right) \frac{6 \pi \varepsilon_{s}}{4 D}\left\langle\phi_{m}\right\rangle\left(x_{||}\right) .
$$

This expression differs from the standard two-fluid model (Eq. 28) in two respects. One is that it allows for an inhomogeneous average bulk fluid temperature field, and the other is that the temperature difference is between the average bulk fluid temperature and the average solid temperature. In order for this to be usable in a two-fluid model, we first need to relate the average bulk fluid temperature to the average fluid temperature. This is easily accomplished by 
Eq. 17 that relates the steady average fluid temperature to the average bulk fluid temperature as $\left\langle\phi^{(f)}\right\rangle\left(x_{\|}\right)=\left\langle\theta^{(f)}\right\rangle\left\langle\phi_{m}\left(x_{\|}\right)\right\rangle$. Now we also assume that the flow is locally fully thermally developed, in which case the heat transfer coefficient $\langle h\rangle\left(x_{\|}\right)$is independent of $x_{\|}$and can be written in terms of the homogeneous average Nusselt number as $\langle h\rangle=k_{f}\langle\mathrm{Nu}\rangle / D$. The resulting expression is a consistent two-fluid model in terms of the average fluid temperature that allows for its inhomogeneous variation:

$$
\left\langle q_{\phi}^{\prime \prime \prime}\right\rangle\left(x_{\|}\right)=\frac{6 \pi \varepsilon_{s} k_{f}\langle\mathrm{Nu}\rangle}{4 D^{2}} \frac{\left\langle\phi^{(f)}\right\rangle\left(x_{\|}\right)}{\left\langle\theta^{(f)}\right\rangle} .
$$

Now although the above expression is in terms of the inhomogeneous average fluid temperature, the two-fluid model assumes that the average fluid temperature is locally homogeneous. Therefore, we recast our model in terms of quantities that are spatially averaged over the domain length $L$ to obtain the following consistent two-fluid model:

$$
q_{c T F-\text { model }}^{\prime \prime \prime}=\frac{6 \pi \varepsilon_{s} k_{f} \mathrm{Nu}}{4 D^{2}\left\langle\theta^{(f)}\right\rangle}\left(\left\langle T^{(s)}\right\rangle-\overline{\left\langle T^{(f)}\right\rangle}\right),
$$

using the expression for the average non-dimensional fluid temperature as

$$
\frac{\overline{\left\langle T^{(f)}\right\rangle}-\left\langle T^{(s)}\right\rangle}{\left\langle T_{m, i n}\right\rangle-\left\langle T^{(s)}\right\rangle} \equiv \frac{1}{L} \int_{0}^{L}\left\langle\phi^{(f)}\right\rangle\left(x_{\|}\right) d x_{\|}=\frac{1}{L} \int_{0}^{L} \frac{\left\langle T^{(f)}\right\rangle\left(x_{\|}\right)-\left\langle T^{(s)}\right\rangle}{\left\langle T_{m, i n}\right\rangle-\left\langle T^{(s)}\right\rangle} d x_{\|},
$$

where the volumetric mean of average fluid temperature is

$$
\overline{\left\langle T^{(f)}\right\rangle}=\frac{1}{L} \int_{0}^{L}\left\langle T^{(f)}\right\rangle\left(x_{\|}\right) d x_{\|} .
$$

Since $\left\langle T^{(f)}\right\rangle$ varies significantly along $x_{\|}$in many cases, the two-fluid definition of $\left\langle T^{(f)}\right\rangle$ in Eq. 28 is appropriately modified using Eq. 56. Therefore, when we compare $q_{T F}^{\prime \prime \prime}$ with PR-DNS data in Section 6, we use the following two-fluid model with average Nusselt number from PR-DNS as

$$
q_{T F}^{\prime \prime \prime}=\frac{6 \varepsilon_{s} k_{f} \mathrm{Nu}}{D^{2}}\left(\left\langle T^{(s)}\right\rangle-\overline{\left\langle T^{(f)}\right\rangle}\right) .
$$

Note that the units of $q_{c T F-\text { model }}^{\prime \prime \prime}$ are $\mathrm{W} / \mathrm{m}^{3}$ whereas the units of $\left\langle q_{\phi}^{\prime \prime \prime}\right\rangle$ are $\mathrm{W} /\left(\mathrm{m}^{3}-\mathrm{K}\right)$. In order to compare PR-DNS data with $q_{c T F-\text { model }}^{\prime \prime \prime}$ and $q_{T F}^{\prime \prime \prime}$, we introduce the average volumetric interphase heat transfer rate from PR-DNS 
$\overline{q_{D N S}^{\prime \prime \prime}}$

$\overline{q_{D N S}^{\prime \prime \prime}}=\frac{1}{L} \int_{0}^{L} q_{D N S}^{\prime \prime \prime}\left(x_{\|}\right) d x_{\|}=\frac{1}{L} \int_{0}^{L}\left(\left\langle T_{m, i n}\right\rangle-\left\langle T^{(s)}\right\rangle\right)\left\langle q_{\phi}^{\prime \prime \prime}\right\rangle\left(x_{\|}\right) d x_{\|}$,

since $q_{D N S}^{\prime \prime \prime}$ can be directly extracted from PR-DNS and its unit is $\mathrm{W} / \mathrm{m}^{3}$. Note that the overbar in all the above equations can be dropped if the average fluid temperature satisfies the criterion of local homogeneity.

\section{Acknowledgments}

This work is partially supported by Department of Energy grant DE-AC0207CH11358 through the Ames Laboratory, Iowa State University. We would like to acknowledge the National Science Foundation for partial support from award CBET 1034307 and CBET 1336941.

\section{Nomenclature}

\section{Upper-case Roman}

$\langle N\rangle \quad$ average number of particles

$\left\langle T_{m, i n}\right\rangle$ average inlet bulk fluid temperature

$\left\langle T_{m, \text { out }}\right\rangle$ average bulk fluid temperature at $x=L$

$\langle\mathbf{W}\rangle \quad$ mean slip velocity

$\langle\mathrm{Nu}\rangle \quad$ average Nusselt number

$\langle P\rangle\left(x_{\|}\right)$average perimeter

$\left\langle T^{(f)}\right\rangle$ average fluid temperature

$\left\langle T^{(s)}\right\rangle$ average solid temperature

$\left\langle T_{m}\right\rangle\left(x_{\|}, t\right)$ the average bulk fluid temperature

$\left\langle\mathrm{Nu}\left(x_{\|}\right)\right\rangle_{M}$ average Nusselt number at axial location $x_{\|}$

$\{\mathrm{Nu}\}_{V}(\omega)$ volume-averaged Nusselt number
$\mathrm{Nu}_{0}$ the limiting value of Nusselt number

$\mathrm{Nu}_{\mathrm{m}} \quad$ Nusselt number for two-fluid model

$\mathrm{Nu}\left(x_{\|} ; \omega\right)$ local Nusselt number

Pr Prandtl number

$\mathrm{Re}_{\mathrm{m}} \quad$ mean slip Reynolds number

A cross-sectional area

$A_{f} \quad$ area occupied by the fluid in a plane perpendicular to the streamwise direction

$A_{s} \quad$ cross-sectional fluid area perpendicular to streamwise direction

convective term at the $n$th time-step

particle diameter

$D_{m} \quad$ grid resolution 
$I_{f}(\mathbf{x}, t)$ the fluid-phase indicator function

$I_{s}(\mathrm{x}, t)$ the solid-phase indicator function

$L \quad$ length of computational domain

$M \quad$ number of realizations

$N_{s} \quad$ number of points at the surface of the sphere

$P\left(x_{\|} ; \omega\right)$ perimeter formed by cutting the particles in the crosssectional plane

$P_{\omega} \quad$ probability for that realization

$R \quad$ sphere radius

$R_{\perp} \quad$ radius of the circle formed by the intersection of the $y-z$ plane with the sphere

$T^{\prime \prime \prime}(f) \quad$ fluctuating components of fluid temperature

$T_{f} \quad$ fluid temperature

$T_{m}\left(x_{\|}, t ; \omega\right)$ bulk fluid temperature for each realization

$T_{s} \quad$ solid temperature

$V \quad$ volume

$V_{f} \quad$ fluid-phase volume

$X \quad$ a random variable uniformly distributed in $(-R, R)$

$X_{c} \quad$ axial coordinate of the sphere center

\section{Lower-case Roman}

$\left\langle q_{\phi}^{\prime \prime \prime}\right\rangle$ average volumetric interphase heat transfer rate per unit tem- perature difference from PRDNS

$\left\langle\mathbf{u}^{(f)}\right\rangle$ the average velocities in the fluid phase

$\left\langle\mathbf{u}^{(s)}\right\rangle$ the average velocities in the solid phase

$\langle h\rangle\left(x_{\|}\right)$inhomogeneous average heat transfer coefficient

$\mathbf{e}_{\|} \quad$ streamwise direction

$\overline{q_{D N S}^{\prime \prime \prime}}$ average volumetric interphase heat transfer rate from PRDNS over axial length

$\triangle x \quad$ grid length

$c_{p f} \quad$ specific heat in the fluid phase

$f_{\phi} \quad$ scalar Immersed Boundary direct forcing in the solid phase

$f_{X} \quad$ probability density function of a random variable uniformly distributed in $(-R, R)$

$k_{f} \quad$ thermal conductivity in the fluid phase

n number density

$n_{j, \perp}^{(s)} \quad$ in-plane component of the outward unit normal vector on the surface of particles

$q_{\perp}^{\prime \prime \prime}\left(x_{\|} ; \omega\right)$ volumetric heat transfer rate per unit temperature difference corresponding to the in-of-plane local interphase heat flux

$q_{\|}^{\prime \prime \prime}\left(x_{\|} ; \omega\right)$ volumetric heat transfer rate per unit temperature difference corresponding to the 


\begin{tabular}{|c|c|c|}
\hline & $\begin{array}{l}\text { out-of-plane local interphase } \\
\text { heat flux }\end{array}$ & $\begin{array}{l}\text { thermal diffusivity in the fluid } \\
\text { phase }\end{array}$ \\
\hline & $\begin{array}{l}\text { out-plane interphase heat flux } \\
\omega) \text { local volumetric interphase }\end{array}$ & $\begin{array}{l}\text { average non-dimensional fluid } \\
\text { temperature }\end{array}$ \\
\hline \multicolumn{2}{|c|}{$\begin{array}{l}q_{\phi}^{\prime \prime \prime}\left(x_{\|} ; \omega\right) \text { local volumetric interphase } \\
\text { heat transfer rate per unit tem- } \\
\text { perature difference }\end{array}$} & $\begin{array}{l}\text { average non-dimensional bulk } \\
\text { fluid temperature }\end{array}$ \\
\hline \multicolumn{2}{|c|}{$\begin{array}{c}q_{C T F-\text { model }}^{\prime \prime \prime} \text { average volumetric inter- } \\
\text { phase heat transfer rate from } \\
\text { improved consistent model }\end{array}$} & $\begin{array}{c}\left\langle\phi_{m}\right\rangle_{\text {out }} \text { average non-dimensional bulk } \\
\text { fluid temperature at } x=L\end{array}$ \\
\hline$q_{D N S}^{\prime \prime \prime}$ & $\begin{array}{l}\text { average volumetric interphase } \\
\text { heat transfer rate from PR- } \\
\text { DNS }\end{array}$ & $\begin{array}{l}\left\langle\psi^{(f)}\right\rangle(\mathbf{x}, t) \text { fluid-phase average } \\
\left\langle\theta^{(f)}\right\rangle \text { average scaled fluid tempera- } \\
\quad \text { ture }\end{array}$ \\
\hline$q_{j, \perp}^{\phi}$ & in-plane interphase heat flux & \multirow{2}{*}{$\begin{array}{c}\left\{\theta^{(f)}\right\}_{V} \text { volumetric mean of the scaled } \\
\text { fluid temperature }\end{array}$} \\
\hline$q_{j}$ & heat flux vector & \\
\hline$q_{j}^{\phi}$ & $\begin{array}{l}\text { heat flux per unit temperature } \\
\text { difference }\end{array}$ & $\begin{array}{l}\text { a realization of particle config- } \\
\text { uration }\end{array}$ \\
\hline \multirow[t]{3}{*}{$q_{T F}^{\prime \prime \prime}$} & average volumetric interphase & $\phi(\mathbf{x}, t)$ non-dimensional temperature \\
\hline & heat transfer rate from two- & fluid density \\
\hline & & scaled fluid temperature \\
\hline \multirow{3}{*}{$\begin{array}{l}r_{h} \\
u_{j}^{\prime \prime}(f)\end{array}$} & heat ratio & \\
\hline & the fluctuating components of & ed porosity \\
\hline & the fluid velocity & solid volume fraction \\
\hline$x_{\|}$ & axial location & Superscripts \\
\hline \multicolumn{2}{|c|}{ Greek Symbols } & $n$th time-step \\
\hline
\end{tabular}

\section{References}

[1] D. J. Gunn, Transfer of heat and mass to particles in fixed and fluidized beds, International Journal of Heat and Mass Transfer 21 (1978) 467-476.

[2] C. Azar, K. Lindgren, E. Larson, K. Mollersten, Carbon capture and storage from fossil fuels and biomass-costs and potential role in stabilizing the atmosphere, Climatic Change 74 (1-3) (2006) 47-79.

[3] L. Shen, M. Zheng, J. Xiao, R. Xiao, A mechanistic investigation of a calcium-based oxygen carrier for chemical looping combustion, Combustion and Flame 154 (3) (2008) 489 - 506. 
[4] J. C. Abanades, E. J. Anthony, D. Y. Lu, C. Salvador, D. Alvarez, Capture of $\mathrm{CO}_{2}$ from combustion gases in a fluidized bed of $\mathrm{CaO}$, Environmental and Energy Engineering 50 (7) (2004) 1614-1622.

[5] C.-K. Yi, S.-H. Jo, Y. Seo, J.-B. Lee, C.-K. Ryu, Continuous operation of the potassium-based dry sorbent $\mathrm{CO}_{2}$ capture process with two fluidizedbed reactors, International Journal of Greenhouse Gas Control 1 (1) (2007) $31-36$.

[6] D. C. Miller, M. Syamlal, R. Cottrell, J. D. Kress, X. Sun, S. Sundaresan, N. V. Sahinidis, S. E. Zitney, D. Bhattacharyya, D. Agarwal, et al., Annual Report: Carbon Capture Simulation Initiative (CCSI) (30 September 2012), 2012.

[7] M. Syamlal, W. Rogers, T. J. O'Brien, MFIX Documentation: Theory Guide., Technical report, National Energy Technology Laboratory, Department of Energy (1993).

[8] B. A. Kashiwa, E. S. Gaffney, Design Basis for CFDLib, Tech. Rep. LAUR-03-1295, Los Alamos National Lab (2003).

[9] J. Sun, F. Battaglia, S. Subramaniam, Hybrid two-fluid dem simulation of gas-solid fluidized beds, Journal of Fluids Engineering 129 (11) (2007) $1394-1403$.

[10] T. B. Anderson, R. Jackson, A fluid mechanical description of fluidized beds, Industrial \& Engineering Chemistry Fundamentals 6 (1967) 527-539.

[11] D. A. Drew, S. L. Passman, Theory of Multicomponent Fluids, Applied Mathematical Sciences, Springer, New York, 1998.

[12] R. Garg, Modeling and simulation of two-phase flows, Ph.D. thesis, Iowa State University (2009).

[13] C. Hrenya, A. Morris, Pachinko revisited: Predicting granular flows and their heat transfer, in: Proceedings of 2014 American Institute of Chemical Engineers Annual Meeting, 2014.

[14] R. Clift, J. R. Grace, M. E. Weber, Bubbles, Drops and Particles, Academic Press, 1978.

[15] J. P. Sorensen, W. E. Stewart, Computation of forced-convection in slow flow through ducts and packed-beds .3. heat and mass-transfer in a simple cubic array of spheres, Chemical Engineering Science 29 (1974) 827-832.

[16] R. Pfeffer, J. Happel, Analytical study of heat and mass transfer in multiparticle systems at low reynolds numbers, AIChE Journal 10 (5) (1964) $605-611$. 
[17] A. Acrivos, E. J. Hinch, D. J. Jeffrey, Heat transfer to a slowly moving fluid from a dilute fixed bed of heated spheres, Journal of Fluid Mechanics 101(2) (1980) 403-421.

[18] D. Kunii, J. M. Smith, Heat transfer characteristics of porous rocks. 2. thermal conductivities of unconsolidated particles with flowing fluids, AIChE Journal 7 (1961) 29-34.

[19] D. Handley, P. J. Heggs, Momentum and heat transfer mechanisms in regular shaped packings, AIAA Journal 46 (1968) 251-264.

[20] H. Littman, R. G. Barile, A. H. Pulsifer, Gas-particle heat transfer coefficients in packed beds at low reynolds numbers, Industrial \& Engineering Chemistry Fundamentals 7 (1968) 554.

[21] D. J. Gunn, J. F. C. Desouza, Heat-transfer and axial dispersion in packedbeds, Chemical Engineering Science 29 (1974) 1363-1371.

[22] J. Shen, S. Kaguei, N. Wakao, Measurements of particle-to-gas heattransfer coefficients from one-shot thermal responses in packed-beds, Chemical Engineering Science 36 (1981) 1283-1286.

[23] N. Wakao, S. Tanisho, B. Shiozawa, Thermal response of packed beds at low Reynolds numbers., Heat Transfer - Japanese Research 6 (4) (1977) 56 -60 .

[24] N. Wakao, S. Kaguei, Heat and mass transfer in packed beds, Vol. 1 of Topics in chemical engineering, Gordon and Breach science, 1982.

[25] R. J. Hill, D. L. Koch, A. J. C. Ladd, The first effects of fluid inertia on flows in ordered and random arrays of spheres, Journal of Fluid Mechanics 448 (2001) 213-241.

[26] R. J. Hill, D. L. Koch, A. J. C. Ladd, Moderate reynolds number flows in ordered and random arrays of spheres, Journal of Fluid Mechanics 448 (2001) 243-278.

[27] M. A. Van der Hoef, R. Beetstra, J. A. M. Kuipers, Lattice-Boltzmann simulations of low-Reynolds-number flow past mono- and bidisperse arrays of sphere: results for the permeability and drag force, Journal of Fluid Mechanics 528 (2005) 233-254.

[28] R. Beetstra, M. A. van der Hoef, J. A. M. Kuipers, Drag force of intermediate Reynolds number flows past mono- and bidisperse- arrays of spheres, AIChE Journal 53 (2007) 489.

[29] X. Yin, S. Sundaresan, Drag law for bidisperse gas-solid suspensions containing equally sized spheres, Industrial and Engineering Chemistry Research 48 (2009) 227-241. 
[30] S. Tenneti, R. Garg, S. Subramaniam, Drag law for monodisperse gasgolid systems using particle-resolved direct numerical simulation of flow past fixed assembiles of spheres, International Journal of Multiphase Flow 37 (9) (2011) 1072-1092.

[31] Z. Yu, X. Shao, A. Wachs, A fictitious domain method for particulate flows with heat transfer, Journal of Computational Physics 217 (2006) 424-452.

[32] Z. G. Feng, E. E. Michaelides, Heat transfer in particulate flows with direct numerical simulation (DNS), International Journal of Heat and Mass Transfer 52 (2009) 777-786.

[33] S. Haeri, J. Shrimpton, A new implicit fictitious domain method for the simulation of flow in complex geometries with heat transfer, Journal of Computational Physics 237 (2013) 21-45.

[34] A. Massol, O. Simonin, T. Poinsot, Steady and unsteady drag and heat transfer in fixed arrays of equal sized spheres, Tech. Rep. TR/CFD/04/13, CERFACS, Toulouse, France (2004).

[35] N. G. Deen, S. H. Kriebitzsch, M. A. van der Hoef, J. A. M. Kuipers, Direct numerical simulation of flow and heat transfer in dense fluid-particle system, Chemical Engineering Science 81 (2012) 329-344.

[36] N. G. Deen, E. Peters, J. T. Padding, J. Kuipers, Review of direct numerical simulation of fluid-particle mass, momentum and heat transfer in dense gassolid flows, Chemical Engineering Science 116 (2014) 710-724.

[37] H. Tavassoli, S. Kriebitzsch, M. van der Hoef, E. Peters, J. Kuipers, Direct numerical simulation of particulate flow with heat transfer, International Journal of Multiphase Flow 57 (2013) 29-37.

[38] Z.-G. Feng, S. G. Musong, Direct numerical simulation of heat and mass transfer of spheres in a fluidized bed, Powder Technology 262 (2014) 62-70.

[39] S. Tenneti, B. Sun, R. Garg, S. Subramaniam, Role of fluid heating in dense gas-solid flow as revealed by particle-resolved direct numerical simulation, International Journal of Heat and Mass Transfer 58 (2013) 471-479.

[40] S. Benyahia, M. Syamlal, T. O'Brien, Summary of MFIX Equations 2012-1, Technical report (2012).

[41] Y.Xu, S. Subramaniam, Effect of particle clusters on carrier flow turbulence: a direct numerical simulation study, Flow, Turbulence and Combustion 85 (2010) 735-761.

[42] M. Tyagi, S. Acharya, Large eddy simulations of flow and heat transfer in rotating ribbed duct flows, Journal of Heat Transfer 127 (2005) 486-498. 
[43] S.Tenneti, S. Subramaniam, Particle-resolved direct numerical simulation for gas-solid flow model development, Annual Review Fluid Mechanics 46 (2014) 199-230.

[44] F. P. Incropera, D. P. DeWitt, T. L. Bergman, A. S. Lavine, Fundamentals of Heat and Mass Transfer, sixth Edition, John Wiley \& Sons Inc, New York, 2006.

[45] S. Tenneti, Momentum, energy and scalar transport in polydisperse gassolid flows using particle-resolved direct numerical simulations, Phd thesis, Iowa State University (2013).

[46] S. Tenneti, R. Garg, C. M. Hrenya, R. O. Fox, S. Subramaniam, Direct numerical simulation of gas-solid suspensions at moderate reynolds number: Quantifying the coupling between hydrodynamic forces and particle velocity fluctuations, Powder Technology 203 (2010) 57-69.

[47] R. Garg, S. Tenneti, J. Mohd-Yusof, S. Subramaniam, Direct numerical simulation of gas-solid flow based on the immersed boundary method, in: S. Pannala, M. Syamlal, T. J. O'Brien (Eds.), Computational Gas-solid Flows and Reacting systems: Theory, Methods and Practice, IGI Global, 2010.

[48] S. V. Patankar, Numerical heat transfer and fluid flow, McGraw-Hill, New York, 1980.

[49] J. M. Yusof, Interaction of massive particles with turbulence, Ph.D. thesis, Cornell University (1996).

[50] B. Sun, S. Tenneti, S. Subramaniam, Characterization of the regime of validity of two-fluid heat transfer models using particle-resolved direct numerical simulation, In preparation.

[51] R. Garg, C. Narayanan, D. Lakehal, S. Subramaniam, Accurate numerical estimation of interphase momentum transfer in Lagrangian-Eulerian simulations of dispersed two-phase flows, International Journal of Multiphase Flow 33 (2007) 1337-1364.

[52] J. Xu, S. B. Pope, Assessment of numerical accuracy of PDF Monte Carlo methods for turbulent reacting flows, Journal of Computational Physics 152 (1) (1999) 192-230.

[53] H. Littman, D. Sliva, Gas-particle heat-transfer coefficients in packed beds at low reynolds numbers, Vol. 7, Proceedings of the Fourth International Heat Transfer Conference, Elsevier, New York, 1970.

[54] J. J. Barker, Heat transfer in packed beds, Industrial and Engineering Chemistry 57 (1965) 43-51. 
[55] Q. Xue, T. J. Heindel, R. Fox, A CFD model for biomass fast pyrolysis in fluidized-bed reactors, Chemical Engineering Science 66(11) (2011) 24402452.

[56] Q. Xue, D. Dalluge, T. J. Heindel, R. O. Fox, R. C. Brown, Experimental validation and CFD modeling study of biomass fast pyrolysis in fluidizedbed reactors, Fuel 97 (2012) 757-769.

[57] M. Mehrabadi, S. Tenneti, S. Subramaniam, Pseudo-turbulent gas-phase velocity fluctuations in homogeneous gas-solid flow: Fixed particle assemblies and freely evolving suspensions, Journal of Fluid Mechanics (Accepted).

[58] R. B. Bird, W. E. Stewart, E. N. Lightfoot, Transport Phenomena, 2nd Edition, John Wiley and Sons, 2002. 